\title{
Multiresolution Molecular Mechanics: Adaptive Analysis
}

Emre Biyikli ${ }^{1}$, Albert C. To ${ }^{1 *}$

${ }^{1}$ Department of Mechanical Engineering and Materials Science \& Center for Simulation and

Modeling, University of Pittsburgh, Pittsburgh, Pennsylvania, USA

*Corresponding Author: Albert C. To, 508 Benedum Hall, University of Pittsburgh,

Pennsylvania 15261, Tel: (412) 624-2052; Email: albertto@pitt.edu 


\begin{abstract}
The concurrent atomistic/continuum coupling method Multiresolution Molecular Mechanics (MMM) has been presented for statics [1] and dynamics [2], its convergence and error structure has been analyzed [3], and a unified and consistent framework for general finite element shape functions has been introduced [4]. The current work presents the adaptivity analysis of the MMM method. To start, the rationale and formulation of the MMM method are briefly introduced. Later, two main features of the adaptivity scheme, refinement and coarsening, are discussed and described in detail and step-by-step procedures are outlined. The adaptivity is tested using three numerical examples: (i) 1-D wave propagation, (ii) 2-D dislocation, and (iii) 3D nanoindentation. The results of numerical examples agree well with those of full atomistic simulations. Furthermore, control parameters of adaptivity schemes offer much flexibility to adjust between accuracy and efficiency. The introduced adaptivity schemes are simple, effective, and accurate owing to the consistency and robustness of MMM.
\end{abstract}

Keywords: Multiscale; molecular dynamics; atomistic/continuum; dynamics; adaptivity 


\section{Introduction}

A class of multiscale methods assemble atomistic and continuum scales together in order to take advantage of both approaches. Continuum is the most traditional and common scale that is analytically and computationally investigated in depth. It assumes that matter completely fills the space it occupies, is continuously distributed, and is infinitely divisible into very small pieces. Owing to the extensive research, continuum theories are well established and thus robust, highly efficient, and accurate; however, they usually fail at the atomistic scale for several reasons. To start with, they ignore the inherent discrete structure of the material, particles such as atoms and electrons. Therefore, continuum approaches leave the physical underpinning of their theory weak [5]. Another reason is the fact that different physical phenomena are in play at each scale; for example, quantum effects are not considered at larger scales but they must be accounted for at the nanoscale [6]. A third reason is because the continuum does not allow the material length to vanish to zero; such as at a crack opening [7]. In rare cases where continuum methods are able to handle the physical phenomena at the atomistic scale, they lack the precision that the atomistic methods are ready to offer. Compared to continuum, atomistic methods are relatively new and require further exploration since their inherent discrete structure does not fit in the more traditional understanding. The most attractive feature of atomistic methods is their high accuracy. For example, the behavior of individual atoms in a friction event at the nanoscale can be tracked in profound detail [8]. In addition, atomistic approaches naturally achieve formation of cracks and other types of defects by breaking and rearrangement of bonds without any additional effort. In spite of these advantages, atomistic methods are computationally very expensive hence limited to small systems. For example, a typical aluminum grain consists of approximately $10^{13}$ atoms, which is much larger than the quantity of atoms a typical computer can handle [9]. For another example, Gracie et al. states that a representative volume element should have a volume of $1000 \mu \mathrm{m}^{3}$, which could be resolved by $8.6 \times 10^{13}$ atoms assuming a lattice constant of $3.6 \AA$ [10]. One of the largest scale simulations is conducted by Abraham et al. in 2002 that includes one billion atoms corresponding to a cube of only 0.3 microns side length [11]. The limitations of computational power is not only restricted to length scale but it is also true for the time scale. For instance, $\mathrm{MD}$ is available to only very high experimental strain rates, i.e., $10^{6}-10^{9} \mathrm{~s}^{-1}$ [12]. 
The main purpose of developing a multiscale method is to systematically reduce the total number of degrees of freedom while preserving accuracy. This way simulations can be run in less time with less computational power. Furthermore, larger length and time scales, which are otherwise unfeasible, can be attained. Reduction in the total number of degrees of freedom is best guided with the following insight: only a small portion of atoms are actually interesting and the rest of the atoms are uninformative [13]. Figure 1 shows a snapshot from a nanoindentation example where only $8 \%$ of the atoms that are physically relevant, hence interesting, are made visible. This particular figure best explains how the multiscale model should be constructed. While the informative atoms can be well investigated by highly accurate atomistic methods, the uninformative and much larger rest of the domain can be handled by efficient continuum methods. In that, the premise is that deformation is free from abrupt changes in the continuum region. This way the entire domain is spatially decomposed into atomistic and continuum regions.

The literature includes many multiscale methods each with its own advantages and disadvantages. Among these methods, the most prominent method is Quasicontinuum (QC) method as first introduced by Tadmot et al. [14]. QC method is essentially built upon two assmputions: Kinematic constraints and energy/force approximation [15-17]. In the former, only a small number of atoms, called representative atoms (rep-atoms), are appointed as degrees of freedom of the system and their positions are explicitly accounted for. Utilizing a mesh, positions of rest of the atoms are interpolated from positions of rep-atoms. The second assumption approximates energy/force by sampling energy/force of particular atoms and multiplying their values in proportion to the number of atoms they represent. The Bridging domain (BD) method couples two decomposed domains of different scales via another domain, called bridging domain, hence the name [18-20]. Two domains of different scales meet at an edge or region where the coupling imposes a displacement constraint in order to achieve compatibility between the two scales. The energy (or Hamiltonian) of the handshake region is a weighted sum of energies of fine and coarse scales. The constraint is imposed by using the augmented Lagrange multiplier method that includes the total energy of the system, the constraint, and a penalty term, which augments the constraint. In the Bridging Scale Method (BSM), the key idea is to divide displacement into orthogonal coarse and fine components [21-23]. The coarse scale components are only the FE displacements interpolated by the FE shape functions. The fine scale components 
are then the difference between the total displacements calculated by the MD simulation and coarse slace components and they are only required in the atomistic region. Two sets of equations of motion are then derived from the coarse and fine components of the displacement. In the coupling of coarse and fine scales, BSM introduces impedance and random forces as specialized boundary conditions on the atomistic region.

The literature also includes the Coupled Atomistic and Discrete Dislocation (CADD) that couples atomistics with linear elastic continuum [24, 25]. A prominent feature of this method is to incorporate dislocations in continuum using the Discrete Dislocation (DD) method in order to reach even larger length scales. The Coupled Atomistic-Continuum (CAC) method combines full atomistic with a coarse-grained finite element framework [26, 27]. In the method, crystalline materials are modeled as continuous collection of lattice cells with a group of discrete atoms inside and governing equations are derived with balance laws. The advantage of the method is its coarse grained model that let elements glide to represent dislocations in the continuum region. The Atomistic to Continuum (AtC) is a force-based concurrent multiscale method that combines atomistics and continuum $[28,29]$ where atomistic (Virial) and continuum stresses (or equivalent forces) are blended over a handshake region. The compatibility is assured through the displacements in a weak sense. In their method, known as the Macroscopic Atomistic Ab initio Dynamics (MAAD), Abraham et al. seamlessly unifies the descriptions of continuum, atomistic, and quantum mechanics [7]. Specifically, they couple the finite element, molecular dynamics, and tight-binding methods, respectively. In a crack propagation problem, for instance, the bond rupture at the crack tip is captured by TB, the nonlinear atom behavior along the crack is captured by MD, and the rest is captured by FEM. Yang et al. proposes force-based Atom Collocation Method (ACM), which is truly meshfree [30]. Implementing a compatible atomistic/continuum interface, the method is proven to be free of ghost forces and provides enhanced adaptation capability by easily turning collocation atoms on and off. Park et al. introduces the Surface Cauchy Born (SCB) energy-based continuum method that efficiently captures the prominent surface effects at the nanoscale by utilizing a carefully modified version of the well-known Cauchy-Born constitutive model around the surfaces [31]. In their method, To et. al. employs Perfectly Matched Layers (PML) at the handshake zone between molecular dynamics and continuum to damp out the high frequency waves that cannot be supported by the 
coarse continuum region [32]. For further information on the literature of multiscale methods, interested readers can see excellent review papers [6, 13, 33-41].

Multiresolution Molecular Mechanics (MMM) is a concurrent energy-based atomistic/continuum coupling multiscale method [1]. The method does not require implementation of a cumbersome treatment over a handshake region. Coarsening is achieved by means of a finite element mesh that is utilized to impose (i) kinematic constraints and (ii) energy approximation. The first one is imposed by using shape functions to interpolate positions of groups of atoms from the nodal positions. The second one is imposed by sampling the energies of groups of atoms by only a few selected atoms. Introducing an atomistic description everywhere in the domain, the method (i) eliminates the requirement of the mesh to be refined to the atomistic resolution, (ii) eliminates the requirement to use pad atoms, (iii) accommodates the continuum to be naturally modeled by atoms without a special procedure, and (iv) mitigates ghost forces to a great extent. Ghost forces are defined to be any residual forces that arise as a result of applying the multiscale method to a configuration that is already in equilibrium with forces equal to zero. The primary reason for the existence of ghost forces is the local/nonlocal mismatch at the atomistic/continuum interface since the former is local and the latter is nonlocal. MMM eliminates this mismatch by employing an atomistic description everywhere in the domain. Likewise, MMM mitigates the effects of wave reflection by using a thermostat in the atomistic region as employed by Curtin et al. [25] as described in the MMM dynamics paper [2]. However, when the wave is the primary concern of a problem, such as shock impact, the wave front is required to be captured by the multiscale method. In reference to this requirement, the capacity of MMM to capture the wave front is demonstrated by solving a 1-D wave problem where the wave is concerted with a full atomistic region. MMM is introduced for statics [1] and dynamics [2] followed by convergence and error structure analysis [3] and introduction of a unified and consistent framework for general finite element shape functions [4]. Although the latter work introduces a general MMM framework for employing shape functions of any order, the current work employs linear elements.

Adaptivity is critical to the development of any concurrent atomistic-continuum coupling method. Considering a moving region of interest such as wave or dislocation, the method can either model the entire path a priori at fine resolution or adaptively refine and coarsen the path. Multiscale methods that lack adaptive features are limited to the first option; they can only be 
employed to solve problems where the path can be guessed a priori and modeling the entire path in fine resolution, which severely limits problem size. Even so, efficiency of the implementation will not be as good as an adaptive method due to overuse of fine resolution. Although many multiscale methods are introduced, only a few of them feature adaptivity. Shenoy et al. [42], Park et al. [43], Kwon et al. [44], Shimokawa et al. [45], and Shan et al. [46] introduced adaptivity to the QC method. Moseley et al. [47], Moseley et al. [48], and Gracie et al. [10] presented adaptive features of the BD method. In addition, Marenic et al. presented review of adaptive methods with a focus on the QC and BD methods [49]. Xiong et al. added adaptivity to the CAC method [27]. Another multiscale method that presented adaptive features is the coarsegrained (CG) method by Praprotnik [50] and Heyden [51]. All these adaptive methods including the current work are empirical in a sense that they are not based on theoretical estimation of errors. In their work, Oden et al. presents rigorous mathematical calculations to serve as a sound basis to estimate the modeling error so that they can control this error adaptively [52]. Another note about adaptive multiscale methods is that the theoretical framework of a concurrent coupling method would dictate how that method performs adaptive analysis, and hence obtained results are still characterized by the theoretical framework rather than the adaptivity scheme.

Adaptivity means automatic tracking of moving regions of interest in order to reduce the number of degrees of freedom that need to be simulated. The moving region of interest can be a wave front in a shock impact problem or a dislocation in a tensile test. Adaptivity automates the tracking of the moving regions of interest that are not known a priori, and thus the simulation is easily setup without much preprocessing. More importantly, adaptivity is crucial for efficiency because it keeps the computationally-expensive fine-resolution regions limited to the regions of interest and prevents overuse. One of the two main functions in adaptive analysis is refinement, which converts coarse regions into fine regions wherever required. Refinement is needed to open the front of the region of interest for it to be able to continue its progress. The second function is coarsening, which converts the fine regions back into coarse regions wherever fine resolution is no longer required. Coarsening is needed to close the moving region of interest so that the target efficiency is retained. Coarsening is especially crucial to keep the method efficient without compromising the accuracy since it would otherwise be too expensive to enlarge the fine regions gradually to everywhere the defects visited. Of course, refinement and coarsening are expected to be activated automatically by the method itself without a need to give a priori instructions. 
The core issue of adaptive analysis lies in defining a criterion to determine whether a region shall be modeled with fine or coarse resolution. One way or another, all criteria try to measure the severity of deformation, which characterizes the regions of interest. These criteria call for refinement of the region when they are satisfied and call for coarsening when they are not. These criteria may depend on potential energy [10, 47, 53], centrosymmetry parameter [54], difference between local and global deformations [12], deformation gradient [46], spatial variation in displacement [16], local shear strain [27], interatomic bonds [47], strain energy density between adjacent nodes and consecutive timesteps [55], or difference between smoothed and exact forces [56]. The refinement criterion is mostly imposed in a way that the atoms or nodes are activated for refinement when their values go out of the prescribed range. In addition, atoms that are too close to the activated atoms are also activated for refinement in some implementations [10, 47, 53]. On the other hand, the criterion for coarsening has the opposite relationship such that it is performed when an atom is not activated for refinement any longer. In that, some implementations impose additional requirements such as they enable coarsening only if the candidate coarse description is accurate enough not to bring in an error more than a prescribed value $[10,42,47,49]$. Similarly, the history of an atom or bond is taken into account such that recently refined entities are excluded from coarsening [47]. Some adaptive techniques perform ad-hoc adjustments of the frequency of calling [10] or tolerance [12] of adaptivity procedures so that they are run frequently enough to capture the relevant physical phenomena.

The adaptive analysis procedures include interpolation and extrapolation of state variables between nodes and atoms. These interpolations are conveniently realized by the finite element shape functions $[48,56]$. The refinement procedure interpolates for the positions, velocities, and in some cases accelerations of the new atoms are activated for refinement. Furthermore, thermal vibrations could be added to preserve temperature of the system in a temperature oriented application. In the case that atoms in the coarse regions are turned off, i.e., not stored in the memory, lattice sites of these atoms can be approximately reproduced [17]. The coarsening can be realized by shape functions or by least squares fit [10].

Adaptive analysis by means of either refinement or coarsening introduces a disturbance to the system, which may result in intolerable error. Through a numerical example, Moseley et al. show that the problem arises from instantaneous conversion of fine regions to coarse regions or vice 
versa [48]. That is why the authors carry out the adaptation procedures in several timesteps instead of only one timestep. Gracie et al. staggers the execution of refinement and coarsening procedures in time to alleviate the error [10]. Miller et al. [57] and Shenoy et al. [42] re-establish equilibrium after each adaptation step since the system is considered to be no longer in equilibrium. In a similar way, Miller et al. limits the adaptive analysis to focus on a particular region or limits the counts of adaptation steps in order to restrict adaptation from becoming unduly costly [57]. It should, however, be noted that coarsening will always introduce some sort of error since it is essentially an approximation of the full atomistic by construction [10].

The coarse regions of multiscale models can be meshed by either a uniform or a graded mesh. The uniform mesh dictates a constant mesh size all over the region while the graded mesh enables variation in mesh size. The graded mesh is particularly important to multiscale methods since it can be utilized to dissipate part of the high frequency waves in the case that the mesh size is reduced down to atomistic resolution near the atomistic/continuum interface. Another benefit of the graded mesh is its capability of reaching very coarse resolution by means of very large elements in the continuum region in order to enhance the efficiency. Despite its benefits, the graded mesh has several significant drawbacks. First of all, generation of a graded mesh is more expensive than generation of a uniform mesh. Second, adaptivity requires a graded mesh to be re-generated every time it executes refinement or coarsening since places of fine and coarse regions are updated. Third, generation of a mesh is followed by building shape functions and atom-element relationships, e.g., designating atoms inside an element. The second and third tasks may become extremely expensive because the costs of these computations are already high and may become even higher with frequent updates. Moreover, some adaptivity procedures are recursively run until equilibrium is achieved [16]. As a result, this iterative procure may be prohibitively expensive. Last, the uniform mesh is also favored for its simplicity in implementation and generation.

In the current work, adaptive analysis of MMM is introduced. In contrast to previous methods that require special atomistic/continuum interface treatment, the underlying MMM framework allows the adaptive analysis to be implemented easily. The presented adaptivity scheme is simple, effective, and accurate as demonstrated by the results of three numerical examples including one-dimensional wave propagation, two-dimensional dislocation, and three- 
dimensional nanoindentation. The scheme employs a uniform mesh over the entire region for its aforementioned benefits. Owing to its consistency and robustness, MMM does not need to employ any special technique to smooth the adverse effects of adaptivity. The paper is organized as the following: rationale and mathematical formulation of the method are introduced in Section 2, adaptivity algorithm and procedures are presented in section 3, descriptions and results of numerical examples are given in Section 4, and conclusions are collected in Section 5.

\section{Formulation}

The Multiscale Molecular Mechanics (MMM) method extends molecular mechanics to larger scales by employing continuum methods. More specifically, molecular mechanics is employed in regions of interest and a continuum approach by means of finite elements is utilized elsewhere. One key feature of MMM is that an atomistic description is used for the entire domain. The atoms are regarded as degrees of freedom in the atomistic region whereas they are represented by some special atoms in the continuum region. These special atoms and atoms in the atomistic region are assigned a type and called "rep-atoms". More specifically, the special rep-atoms in the continuum region are called interpolating rep-atoms (red atoms in Fig. 2). Setting the interpolating rep-atoms as nodes of a mesh, the continuum region is discretized by finite elements. The mesh, coupled with finite element shape functions, represents the collective behavior of atoms in the continuum region. The atoms that are represented by interpolating repatoms are altogether called ghost atoms and further assigned three types as will be detailed later. The most prominent profit of resolving the entire domain with an atomistic description is having a truly seamless connection between atomistic and continuum regions. Also, atomistic description preserves the essential advantages of full atomistic simulations, which are otherwise

eliminated by completely continuum descriptions as they lost stochasticity in the CAC method [26]. The price paid in using the atomistic description is the computational cost of storing and computing for the crowded number of atoms. The number of ghost atoms can be potentially reduced by excluding inactive atoms from storage and calculations.

A key point in the MMM framework lies in an assumption it makes in the continuum region. MMM assumes that deformation of the continuum region is linear; therefore, it can be 
represented by linear elements. As noted earlier, MMM is originally introduced using linear elements [1] and then extended to higher order elements [4]; but we are considering linear element in the current work. In correspondance to linear elements, the strain (or the deformation gradient) in each element is constant. The assumption is further supported by the fact that the difference between true and homogeneous deformation is minimized within the cutoff radius of the potential. As a result of this assumption, the energy distribution in an element is constant for the interior atoms. That is, the atoms that do not have neighbors in other elements have the same energy. This useful property still holds after the element is deformed. The uniformity of energy distribution motivates energy sampling, which is a shortcut to compute the energies of atoms in an element. Energy of a preselected atom is sampled and value of the energy is assigned to other atoms in the element. The atom that is used to sample the energy is called the "primary sampling atom" (blue atom in Fig. 2) and the atoms whose energies are sampled by the primary sampling atom are called the "non-sampling atoms" (gray atoms in Fig. 2). The energy sampling scheme saves the computational cost of calculating energies of the majority of atoms in the continuum region.

In accordance with the earlier assumption, MMM employs kinematic constraints in the continuum region. That is, the positions of atoms that are not appointed as degrees of freedom are interpolated from the positions of interpolating rep-atoms located at the nodes of the mesh. In order to be consistent with the linear elements, MMM employs linear shape functions in the continuum region [1]. The shape functions are utilized not only for interpolating the positions of atoms but also for interpolation of the masses, velocities, and other state variables since the repatoms are meant to fully represent other atoms. The shape functions utilized in MMM are built and operated in the same way as in FEM. For instance, an interpolating rep-atom is locally supported by the corresponding shape functions of the elements where it is a node by construction.

Of importance is that the uniformity of energy distribution in an element is only valid in the interior of the element. This is because the energy of the atoms along the edges of an element depend on the atoms in neighbor atoms located in adjacent elements. This asymmetry implies that the edge atoms have different environment and hence different energy than the interior atoms. In order to solve this issue, the energies of some of the atoms should be accounted for 
individually instead of employing the earlier one-for-all sampling relationship for the primary sampling atoms. These atoms are called the "secondary-sampling atoms" (green atoms in Fig. 2). They are utilized near the edges for the obvious reasons and around the rep-atoms for better sampling. Consequently, MMM has five types of atoms as listed in Table 1.

Four out of five types of atoms are described above. The fifth type of atom is called the "noninterpolating rep atom" (black atoms in Fig. 2), which is identical to an atom in full atomistic. These atoms are employed in atomistic regions of the domain in order to obtain high accuracy. In total, MMM has five types of atoms each for a specific purpose. Nonetheless, atoms can be assigned to any of these types, thus providing the method with a high level of flexibility. For instance, primary sampling atoms and secondary sampling atoms can be chosen in any number and location in an element, and suggestions on this matter have been recently proposed [4]. This flexibility provides the user with an opportunity to tune the atom type selection for the optimum balance between accuracy and efiicicnecy. MMM is first introduced in its statics form, called MMS, [1] and then extended to zero temperature dynamics, called MMD, [2]. In addition, convergence and error analysis of the method is presented [3]. Yang et al. presented the analysis on different sampling schemes that, in turn, concluded with suggestions for the most optimum schemes [4].

In the following, the concepts described above will be cast into the mathematical formulation of the MMD method. First of all, we define the linear shape functions $\boldsymbol{N}$ used in standard FE methodology in order to accommodate reduced degrees of freedom of the original system. Notations required to establish the mathematical formulation of MMD are given next. $N_{r}, N_{g}$, $N_{p s a}, N_{s s a}$ are the set of rep-atoms, ghost atoms, primary sampling atoms, and secondary sampling atoms, respectively. Rep-atoms are further divided into two types: interpolating rep-atoms $\left(N_{\text {irep }}\right)$ and non-interpolating rep-atoms $\left(N_{\text {nirep }}\right)$, hence $N_{r}=N_{\text {irep }}+N_{\text {nirep. }}$ Let $\mathbf{r}^{N_{r}}=\left(\mathbf{r}_{\mathrm{r} 1}, \mathbf{r}_{\mathrm{r} 2}, \ldots, \mathbf{r}_{N_{r}}\right)$ denote the set of coordinates of rep-atoms, $\mathbf{r}^{N_{g}}=\left(\mathbf{r}_{\mathrm{g} 1}, \mathbf{r}_{\mathrm{g} 2}, \ldots, \mathbf{r}_{N_{g}}\right)$ ghost atoms, $\mathbf{r}^{N_{\text {irep }}}=$ $\left(\mathbf{r}_{\text {irep } 1}, \mathbf{r}_{\text {irep2 }}, \ldots, \mathbf{r}_{N_{\text {irep }}}\right)$ interpolating atoms, and $\mathbf{r}^{N_{\text {nirep }}}=\left(\mathbf{r}_{\text {nirep } 1}, \mathbf{r}_{\text {nirep } 2}, \ldots, \mathbf{r}_{N_{\text {nirep }}}\right)$ noninterpolating rep-atoms. Thus, we have $\mathbf{r}^{N_{A}}=\left(\mathbf{r}^{N_{r}}, \mathbf{r}^{N_{g}}\right), \mathbf{r}^{N_{r}}=\left(\mathbf{r}^{N_{\text {irep }}}, \mathbf{r}^{N_{\text {nirep }}}\right)$, and $\mathbf{r}_{\alpha}=$ $\mathbf{r}_{\alpha 0}+\mathbf{u}_{\alpha}, \forall \alpha \in N_{A}$ where $\mathbf{r}_{\alpha 0}$ denote the initial coordinates of atom $\alpha$. In addition, the shape function $\boldsymbol{\aleph}_{\gamma}$ of a rep-atom $\gamma$ is defined as follows: if $\gamma$ represents an interpolating rep-atom, then 
$\aleph_{\gamma}$ is the standard interpolation shape function; if $\gamma$ represents a non-interpolating rep-atom, then $\aleph_{\gamma}$ is unity at itself and is zero at all other atoms. Note that $\aleph_{\gamma}$ is evaluated at the atom positions. Then, coordinates and velocities of the ghost atoms can be interpolated through the following expression as

$$
\begin{gathered}
\mathbf{r}_{\beta}=\mathbf{r}_{\beta 0}+\mathbf{u}_{\beta}=\mathbf{r}_{\beta 0}+\sum_{\gamma \in N_{r}}^{N_{r}} \boldsymbol{\aleph}_{\gamma}\left(\mathbf{X}=\mathbf{r}_{\beta 0}\right) \mathbf{u}_{\gamma}, \forall \beta \in N_{g} \\
\dot{\mathbf{r}}_{\beta}=\sum_{\gamma \in N_{r}}^{N_{r}} \boldsymbol{\aleph}_{\gamma}\left(\mathbf{X}=\mathbf{r}_{\beta 0}\right) \dot{\mathbf{r}}_{\gamma}, \forall \beta \in N_{g}
\end{gathered}
$$

Coordinates and velocities of the ghost atoms depend only on the coordinates and velocities of interpolating rep-atoms, therefore $\mathbf{r}^{N_{g}}$ and $\dot{\mathbf{r}}^{N_{g}}$ can be expressed as a function of $\mathbf{r}^{N_{r}}$ as follows

$$
\begin{aligned}
& \mathbf{r}^{N_{g}}=\Psi\left(\mathbf{r}^{N_{r}}\right) \\
& \dot{\mathbf{r}}^{N_{g}}=\Phi\left(\dot{\mathbf{r}}^{N_{r}}\right)
\end{aligned}
$$

Then, total potential energy of the original system is approximated as follows [1]

$\widehat{\Pi}\left(\mathbf{r}^{N_{r}}\right)=\sum_{i}^{N_{r}} E_{i}\left(\mathbf{r}^{N_{r}}, \Psi\left(\mathbf{r}^{N_{r}}\right)\right)+\sum_{j}^{N_{p s a}} w_{j} E_{j}\left(\mathbf{r}^{N_{r}}, \Psi\left(\mathbf{r}^{N_{r}}\right)\right)+\sum_{k}^{N_{s s a}} E_{k}\left(\mathbf{r}^{N_{r}}, \Psi\left(\mathbf{r}^{N_{r}}\right)-\right.$

$\sum_{\alpha=1}^{N_{A}} \mathbf{f}_{\alpha}^{e x t} \mathbf{u}_{\alpha}\left(\mathbf{r}^{N_{r}}\right)$

where $w_{j}$ is the weight associated with the primary sampling atom in element $j$. Let $N_{g}^{e_{j}}$ and $N_{s s a}^{e_{j}}$ denote the set of ghost atoms and secondary sampling atoms in element $e_{j}$, respectively. Assuming one primary sampling atom is employed in $e_{j}$, we have

$$
w_{j}=\left|N_{g}^{e_{j}} \backslash N_{s s a}^{e_{j}}\right|
$$

which is the number of ghost atoms that are not secondary sampling atoms in element $j$. In other words, $w_{j}$ is the sum of numbers of primary sampling and non-sampling atoms in element $j$.

Let $\mathbf{p}^{N_{r}}=\left(\mathbf{p}_{\mathrm{r} 1}, \mathbf{p}_{\mathrm{r} 2}, \ldots, \mathbf{p}_{N_{r}}\right)$ denote the set of momenta of rep-atoms. The momentum of an atom $\alpha$ of the original system can then be approximated as follows

$$
\widehat{\mathbf{p}}_{\alpha}\left(\mathbf{p}^{N_{r}}\right)=m_{\alpha} \cdot \sum_{\gamma \in N_{r}}^{N_{r}} \boldsymbol{\aleph}_{\gamma}\left(\mathbf{X}=\mathbf{r}_{\beta 0}\right) \dot{\mathbf{r}}_{\gamma}=m_{\alpha} \cdot \sum_{\gamma \in N_{r}}^{N_{r}} \boldsymbol{\aleph}_{\gamma}\left(\mathbf{X}=\mathbf{r}_{\alpha 0}\right) \frac{\mathbf{p}_{\gamma}}{m_{\gamma}}
$$

Then, the total kinetic energy of the original system can be approximated as a function of $\mathbf{p}^{N_{r}}$ 


$$
\widehat{T}\left(\mathbf{p}^{N_{r}}\right)=\sum_{\alpha=1}^{N_{A}} \widehat{T}_{\alpha}\left(\dot{\mathbf{r}}^{N_{r}}\right)=\sum_{\alpha=1}^{N_{A}} \frac{1}{2 m_{\alpha}} \widehat{\mathbf{p}}_{\boldsymbol{\alpha}}\left(\mathbf{p}^{N_{r}}\right) \cdot \widehat{\mathbf{p}}_{\boldsymbol{\alpha}}\left(\mathbf{p}^{N_{r}}\right)
$$

With the above expression in hand, total Hamiltonian of the MMD system can be written as

$$
\widehat{H}\left(\mathbf{r}^{N_{r}}(t), \mathbf{p}^{N_{r}}(t)\right)=\widehat{T}\left(\mathbf{p}^{N_{r}}\right)+\widehat{\Pi}\left(\mathbf{r}^{N_{r}}\right)
$$

then the approximated Hamiltonian canonical equations of motion are

$$
\begin{aligned}
& \dot{\mathbf{p}}_{\gamma}=-\frac{\partial \widehat{H}}{\partial \hat{\mathbf{r}}_{\gamma}} \\
& \dot{\mathbf{r}}_{\gamma}=-\frac{\partial \widehat{H}}{\partial \mathbf{p}_{\gamma}}
\end{aligned}
$$

for $\forall \gamma \in N_{r}$. Equations (11) and (12) can be combined to yield

$$
\mathbf{M} \ddot{\mathbf{r}}^{N_{r}}=-\frac{\partial \widehat{\Pi}\left(\mathbf{r}^{N_{r}}\right)}{\partial \mathbf{r}^{N_{r}}}=\mathbf{f}^{i n t}+\mathbf{f}^{\text {ext }}
$$

where the mass matrix $\mathbf{M}$ and interatomic force vector $\mathbf{f}^{\text {int }}$ are given as follows, respectively

$$
\begin{aligned}
& \mathbf{M}=\sum_{\alpha=1}^{N_{A}} m_{\alpha}\left(\aleph^{N_{r}}\right)^{\mathbf{T}} \boldsymbol{\aleph}^{N_{r}}=\left[\begin{array}{ccccc}
\sum_{\alpha=1}^{N_{A}} m_{\alpha} \aleph_{r 1} \aleph_{r 1} & \sum_{\alpha=1}^{N_{A}} m_{\alpha} \aleph_{r 2} \aleph_{r 1} & \ldots & \sum_{\alpha=1}^{N_{A}} m_{\alpha} \aleph_{N_{r}} \aleph_{r 1} \\
\sum_{\alpha=1}^{N_{A}} m_{\alpha} \aleph_{r 1} \aleph_{r 2} & \sum_{\alpha=1}^{N_{A}} m_{\alpha} \aleph_{r 2} \aleph_{r 2} & \ldots & \sum_{\alpha=1}^{N_{A}} m_{\alpha} \aleph_{N_{r}} \aleph_{r 2} \\
\vdots & \vdots & \vdots & \vdots \\
\sum_{\alpha=1}^{N_{A}} m_{\alpha} \aleph_{r 1} \aleph_{N_{r}} & \sum_{\alpha=1}^{N_{A}} m_{\alpha} \aleph_{r 2} \aleph_{N_{r}} & \ldots & \sum_{\alpha=1}^{N_{A}} m_{\alpha} \aleph_{N_{r}} \aleph_{N_{r}}
\end{array}\right]
\end{aligned}
$$

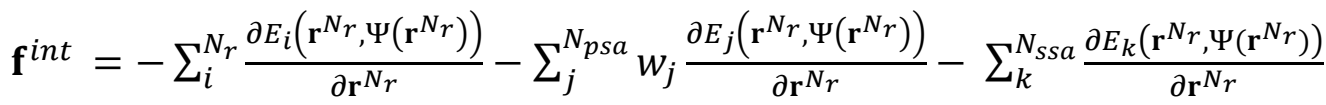

where $\aleph^{N_{r}}=\left(\aleph_{\mathrm{r} 1}, \aleph_{\mathrm{r} 2}, \ldots, \aleph_{N_{r}}\right)$ is the set of interpolation shape functions.

Before closing this section, several remarks are in the order. First, the mass matrix in equation (14) is diagonally lumped for the sake of efficiency. Second, the Berendsen thermostat [58] is applied in order to regulate temperature of the system. The details of mass lumping and thermostat can be found in [2]. Third, it should be noted that MMD is currently a zero temperature dynamics method and it is left as a future work to extend it to finite temperature. Therefore, presented systems are highly damped and their dynamics are limited. 


\section{Adaptivity}

In this section, the adaptivity scheme of MMM is described in detail and refinement and coarsening procedures are outlined. The adaptivity criteria of MMM are based on potential energy, radial influence, and element integrity. First, potential energy is a good indicator of distortion and it is already available $[10,47,53]$. A threshold value is input to the simulation such that atoms with higher potential energies are activated for refinement. According to this criterion, an atom $i$ is activated when

$$
E_{i} \geq E_{\text {threshold }}
$$

where $E_{i}$ is the potential energy of atom $i, E_{\text {threshold }}$ is the input threshold value. Second, atoms within an input radius of activated atoms are also activated for refinement in order to include the region that may possibly be influenced by the distortion $[10,47,53]$. This way, it is made sure to refine the regions that may be directly or indirectly affected by the ongoing physical phenomena. Third, all of the atoms inside the elements that include activated atoms are also activated for refinement for the sake of element integrity. In other words, an element is either in the coarse or fine region completely. Of course, only the ghost atoms among the activated atoms are selected and then converted to non-interpolating rep-atoms. Activated atoms are assigned with only velocities since their positions are already available thanks to the atomic description. That is, current positions of atoms are readily used without any need for a special procedure, which also holds for coarsening. The velocities are interpolated from the corresponding interpolating repatoms with respect to shape functions using Equation (2). In order to conserve mass, the masses of activated atoms take on their original values and those of the corresponding interpolating repatoms are decreased in proportion to reduction of the number of ghost atoms they represent. Owing to the consistency and robustness of MMM, the adaptivity scheme does not require any special smoothing technique as noted in the introduction section. It should be noted that MMM

employs a uniform mesh over the entire region for its aforementioned benefits. The adaptivity function is called periodically with respect to an input number of iterations. In total, adaptivity is controlled by three parameters: (i) Threshold of the potential energy, (ii) radius of influence, and (iii) frequency of update. The refinement procedure is outlined in Table 2. 
The coarsening procedure is opposite to the refinement procedure. In order for an element that is currently full of non-interpolating rep-atoms to be coarsened, none of the rep-atoms must be activated for refinement. Only then is the coarsening activated and all atoms inside the element are assigned with the types that the MMM scheme specifies. The positions of atoms are left as is and new shape functions are constructed. This way, the element is able to preserve its distorted configuration in order to keep peace with its environment. Otherwise, the original shape functions would dictate the original configuration that may not fit to the current environment and cause instability. The velocities and masses of the ghost atoms are extrapolated to the corresponding interpolating rep-atoms where the former extrapolation is performed by shape functions using Equation (2). The latter extrapolation is carried out by equally distributing the total mass of atoms in the element to the interpolating rep-atoms. For example, an element having $N$ atoms of mass of $M$ in it has a total mass of $N x M$. In coarsening, say there are $I$ interpolating rep-atoms in the element, a mass of $N x M / I$ is distributed to each interpolating-rep atom. These extrapolations are carried out in order to conserve mass and momentum of the element and, in turn, the system. The coarsening procedure is outlined in Table 3.

It is a well-known fact that forces derived from a global energy functional conserve linear momentum, angular momentum, and total energy. In addition, the conservation of mass is assured by construction. Analytical proofs to these conservation properties of the MMM method are beyond the scope of this work. After the analytical construction of a method, there are numerical considerations that violate the conservation laws. First is the introduction of a cutoff radius that truncates the interactions beyond a few neighbor shells $[41,59,60]$. Neighbor atoms travelling in and out of this borderline may have their properties accounted for in an on-and-off manner, which would violate the conservation laws. Second, MMM employs a velocity Verlet time integration scheme that is proven to be non-conservative but symplectic, which means showing nearly conservative behavior [41]. The third property is the use of a thermostat to regulate the temperature of the system [61]. A thermostat achieves this regulation by including additional forces on atoms to impose an external force on the system. In spite of these inaccuracies, refinement and coarsening procedures are carefully tailored to conserve mass and momentums of the system. However, the energy cannot be exactly conserved due to inclusion 
and exclusion of energy approximation in refinement and coarsening, respectively [47]. After all, our own numerical investigations on simple models conclude that the method shows nearly conservative behavior.

\section{Numerical examples}

The following numerical examples have been conceived to demonstrate the validity and accuracy of MMM, which is achieved by comparing MMM results to the full atomistics ones where the latter served as a datum. When the trajectories are being compared, it should be noted that the energy functional is highly non-convex and has many metastable configurations at local minima. Due to this characteristic property, many deformation paths are possible and hence deformation behavior of the crystals lacks uniqueness $[16,26]$. Another matter is that results of dynamic simulations oscillate about some mean value due to inherent vibration of atoms. For the sake of clarity, forces in the presented results are averaged from a large group of atoms over an interval of time. In the adaptivity scheme, all the considered models start as a fully coarse model but they are automatically refined at the very beginning of the simulation, for instance, due to a wave or surface effects.

The proposed method is tested for three numerical examples: (i) one-dimensional (1-D) wave propagation; (ii) two-dimensional (2-D) dislocation; and (iii) three-dimensional (3-D) nanoindentation. The iterations are integrated by the Velocity-Verlet scheme. The interactions are truncated beyond the second nearest neighbor. All simulations are run with our home-grown $\mathrm{C}++$ code that is benchmarked against LAMMPS [62]. Post-processing, trajectories of the 1-D wave propagation, and quantitative analysis are conducted by MATLAB. Trajectories of the 2-D dislocation and 3-D nanoindentation are rendered by the fast and user-friendly visualization software OVITO [63].

\subsection{1-D Wave Propagation}

The first numerical example is a 1-D wave propagation that is simulated to demonstrate the capability of the method to track wave motion. The model considered here is consisted of a chain of 2405 atoms. The atoms are separated by $1 \AA$ in order to speed up propagation of the wave. 
The model is fixed by two atoms at both ends (Fig. 3). The MMD model is coarsened by a mesh of elements of size 100ro, which included one primary sampling atom in the center and four secondary sampling atoms around the interpolating rep-atoms. The interactions are modeled with the Lennard-Jones potential with parameters $\sigma=1 \AA$ and $\varepsilon=1 \mathrm{eV}$ and mass is set to $1 \mathrm{~g} / \mathrm{mole}$. The adaptivity criteria are set to $-0.0555 \mathrm{eV}$ ( $93 \%$ of the initial energy value) potential energy threshold, $150 \AA$ radius of influence, and 50 iterations of update frequency. As discussed later, the first criterion (i.e., potential energy threshold) is adjusted by the user depending on which measure is prioritized: accuracy or efficiency. The latter criteria can be adjusted in a similar fashion so that larger radius of influence or smaller update frequency results with higher accuracy but lower efficiency and vice versa. All three adaptivity criteria in this and other examples are adjusted in an ad-hoc fashion in order to produce good results.

A Gaussian wave including both high and low frequency components is introduced into the center of the model by Eq. (17), see right half of it in Figure 4.

$$
u(x, t=0)=\left\{\begin{array}{c}
A \frac{e^{-(x / \sigma)^{2}}-u_{c}}{1-u_{c}}\left[1+b \cos \left(\frac{2 \pi x}{H}\right)\right],|x| \leq L_{c} \\
0,|x|>L_{c}
\end{array}\right.
$$

where $\sigma=20, H=\sigma / 4, A=0.01, b=0.2, L_{c}=4 \sigma$, and $u_{c}=0$.

The simulations are run for $500 \mathrm{fs}$ at a timestep of $0.1 \mathrm{fs}$. At the beginning of the simulations, the wave splits into two parts where they travel opposite to each other towards the ends. As the adaptivity adds and removes rep-atoms, the overall ratio of rep-atoms starts at $34 \%$, increases to $67 \%$, and ends at $51 \%$. Figure 5 demonstrates trajectories of wave propagation for full atomistic (a, c, e) and MMD (b, d, f). The results are indistinguishable at any step of the simulation. In addition, the kinetic energy of a region as marked in Figure 6 is monitored during the course of the simulations. The region consists of an element with 101 atoms and the kinetic energy is computed as sum of the kinetic energies of atoms (Fig. 7). The kinetic energy slightly increases as low frequency component of the wave enters into the region and then decreases as it starts to leave. At this point, the kinetic energy sharply increases as high frequency components of the wave enter and then sharply decreases upon their leave. The kinetic energies of the full atomistic and MMD being monitored are again indistinguishable as also supported by the relative difference. 


\subsection{2-D Dislocation}

The second numerical example is a 2-D dislocation that is simulated to demonstrate the capability of the adaptivity to consistently refine and coarsen as a defect migrates. The $2 \mathrm{D}$ model is consisted of a rectangular plate of 21,709 atoms in a hexagonal configuration. The atoms are separated by the equilibrium distance of the Morse potential and then initially relaxed by static energy minimization. The models are fixed by two layers of atoms at the bottom end (Fig. 8 left). The MMD model is coarsened by a mesh of triangular elements of size 20ro, which included one primary sampling atom in the center and secondary sampling atoms around the interpolating rep-atoms (Fig. 8 - right). The interactions are modeled with the Morse potential with parameters $D_{0}=0.5093 \mathrm{eV}, \alpha=1.45731 / \AA$, and $r_{0}=2.58 \AA$ and mass is set to 26.9815 $\mathrm{g} / \mathrm{mole}$. The adaptivity criteria are set to $-1.6 \mathrm{eV}$ ( $91 \%$ of the initial energy value) potential energy threshold, $10 \AA$ radius of influence, and 100 iterations of update frequency. Temperature of the system is kept constant at $1 \mathrm{~K}$ by the Berendsen thermostat. The models are cropped at their upper-left corners in order to foster dislocation nucleation.

The simulations are run for $1.2 \mathrm{~ns}$ at a timestep of $0.5 \mathrm{fs}$. Two layers of atoms from top of the system are pulled apart at a constant speed corresponding to $10^{-8} \mathrm{~s}^{-1}$ strain rate. As the adaptive MMD method adds and removes rep-atoms, the overall ratio of rep-atoms fluctuated around $27 \pm 3 \%$. Figure 9 demonstrates trajectories of dislocation glide for full atomistics and MMD where only the atoms with higher energies are highlighted in the left and center panels. The snapshots are taken from different timesteps of full atomistic and MMD to emphasize the reaction of models to dislocation glide. The latency between the two models is apparent from the stress-strain curve as presented in Figure 10. The force in Figure 10 is calculated by averaging the $y$-components of forces of the fixed atoms, while the strain is obtained from averaging $y$ components of the distance between fixed atoms. Until the yield point, where the dislocation nucleates, stress-strain curves match perfectly, which is attributed to the uniformity of the deformation. Later on, two differences are observed: (i) nucleation of dislocation is predicted earlier by the MMD model and (ii) the burst in the stress-strain curve of the full atomistic model is recorded stronger. These differences are attributed to the constraining effects of the limited 
size of the atomistic region [29]. Nevertheless, the burst in the stress-strain curve can be further explained with the fact that, although the deformation mechanisms (i.e., perfect edge dislocation) of the two models are the same, energies of dislocations are different. The energy of a dislocation is sum of its nearby core energy and its far field elastic energy where the former is a small fraction of the latter [64]. The elastic energy decays only by $1 / r$ where $r$ is the distance from the dislocation core. Therefore, it may be the case that the long range distribution of the elastic energy is not accurately covered by the confined atomistic region of the MMD model around the dislocation core. The results could be made more accurate by using a larger radius of influence and enlarging the high resolution region, but this is a matter of accuracy versus efficiency depending on the user's preference. In addition, the difference in the energy of dislocations can be inferred from the stress-strain curves since the stronger burst is related to a higher decrease in the strain energy of the system. In agreement, a larger number of atoms is highlighted in Fig. 9 since more atoms with higher energies are involved in the dislocation of the full atomistic model. It may also be possible that the difference in the nucleation stresses is due to the radius of the influence used to capture the onset of nucleation was too small for the present stress-stress gradient since the image stress acting on a dislocation tends to infinity as the dislocation approaches a surface in linear elasticity. These discussions are subject of further research; and, as a matter of fact, the purpose of developing the presented multiscale method is to provide a tool to study such phenomena. After annihilation of the dislocations, both the full atomistic and MMD models continue to harden in a similar fashion. Overall, the trajectories and stress-strain curves agree well and the adaptivity successfully tracks and captures the dislocation glide.

\subsection{3-D Nanoindentation}

The third numerical example is a 3-D nanoindentation is simulated to demonstrate the capability of the adaptivity to capture large amount of dislocations and stacking faults nested together. The model is consisted of a rectangular prism of 78,033 atoms in FCC configuration. The atoms are separated by equilibrium distance given by the potential and then initially relaxed by both static energy minimization and dynamics. The models are fixed by two layers of atoms at bottom ends in all directions and they are fixed by two layers of atoms at the lateral faces in normal directions 
(Fig. 11 - left). The MMD model is coarsened by tetrahedral elements of size 12ro, which consists of secondary sampling atoms (Fig. 11 - right). The interactions are modeled with the Morse potential with parameters $D_{0}=0.2703 \mathrm{eV}, \alpha=1.16461 / \AA$, and $r_{0}=3.253 \AA$ and mass is set to $26.9815 \mathrm{~g} / \mathrm{mole}$. The adaptivity criteria are set to -2.47 ( $97 \%$ of the initial energy value) and $-2.50 \mathrm{eV}$ ( $98 \%$ of the initial energy value) potential energy threshold, $2 \AA$ radius of influence, and 100 iterations of update frequency. Temperature of the system is kept constant at $1 \mathrm{~K}$ by the Berendsen thermostat. The simulations are run for $300 \mathrm{ps}$ at a timestep of $0.5 \mathrm{fs}$. An indenter is pushed towards the top surface with respect to Eq. (18).

$$
F(r)=-K(r-R)^{3}
$$

where $K$ is $10 \mathrm{eV} / \AA^{2}, R$ is $80 \AA$, and $r$ is the distance between the atom and the indenter. The indenter is pushed constantly at a speed of $50 \AA / \mathrm{ns}$.

As the adaptivity adds and removes rep-atoms, the overall ratio of rep-atoms reaches $54 \%$ and $75 \%$ for MMD models with criterion $-2.47 \mathrm{eV}$ and $-2.59 \mathrm{eV}$, respectively. Figure 12 demonstrates trajectories at the onset of plastic deformation for full atomistic and MMD. The similarity of the deformation paths proves the success of MMD models. Figure 13 demonstrates distorted atoms at a later stage of plastic deformation for full atomistic and MMD models. The atoms are colored with respect to deformation types utilizing centrosymmetry parameter [65]. Since it is difficult to distinguish the individual defects and decide if MMD models are successful, a statistical comparison is given by means of distribution of the centrosymmetry parameter in Figure 14. The agreement between the statistical distributions suggests that the MMD model captures various types of defects well. In addition, the force versus depth curves are given in Figure 15. The forces are calculated from the total forces on the indenter and depth is calculated from the displacement of the indenter. The curve obtained from the MMD model with criterion $-2.50 \mathrm{eV}$ performs better and closely captures the various undulations along the curve. The solutions by MMD models with different criteria came out different (Fig. 16).

As expected, the MMD model with a more stringent criterion (i.e., -2.50) uses more rep-atoms and the results are closer to the full atomistic solution. In contrast, the MMD model with a less stringent criterion (i.e., $-2.47 \mathrm{eV}$ ) uses fewer rep-atoms hence the efficiency is higher. This tradeoff shows that the adaptivity criterion can be adjusted to the preference of the user. A second 
trade-off is due to different MMM schemes. The scheme employed in the 3-D nanoindentation example is more expensive and more accurate as compared to the scheme employed in the 2-D dislocation example where the former uses all secondary sampling atoms inside the elements. As a result, the results of the 3-D nanoindentation (especially with criterion -2.50) agree better with full atomistic as can be seen from the force versus depth curve (Fig. 15) and force versus strain curve (Fig. 10). A third trade-off stems from the mesh size. Size of the elements can be set to smaller values to reduce the number of activated atoms due to element integrity criterion. On the contrary, the number of rep-atoms increases as a result of larger number of elements. It is a matter of a few trials and errors to determine the element sizes that would result in better accuracy and efficiency. The authors have experienced little difficulty in tuning an appropriate mesh size for the presented examples.

\section{Conclusions}

In this work, the adaptive MMD is introduced, discussed, and applied to three numerical examples of 1-D wave propagation, 2-D dislocation, and 3-D nanoindentation. A brief review of some recent efforts about method development and adaptivity in the literature provided context for our approach. The rationale and scheme of the adaptivity algorithms and procedures for refinement and coarsening are described in details. The adaptivity criteria are based on potential energy, radial influence, and element integrity. That is, essentially, atoms with high potential energies and their neighbors in close proximity are activated for refinement whereas atoms with neither of these are activated for coarsening. Based on these criteria, the elements are periodically refined or coarsened with respect to the outlined procedures. The adaptivity scheme is simple, effective, and accurate as demonstrated by the results of numerical examples. In comparison of both the qualitative and quantitative results, they are found to agree well with the full atomistic results. Although the 3-D nanoindentation example consists of the most complicated system and includes the largest amount of deformation among other examples, the 2-D dislocation example truly presents the fullest potential of adaptivity since the number of repatoms is kept constant by refinement and coarsening as the deformation mechanism is closely captured. Introduction of the adaptive feature has elevated the MMD method to a level where it is able to handle more complicated problems, albeit at a slow pace. It is difficult to comment on 
the efficiency of the presented MMM models since the primary emphasis of the current work is on adaptivity. The size of the presented MMM models are too small to show satisfactory reduction in the number of degrees of freedom. Also, the current MMM software is designed for flexibility rather than performance for the sake of implementing new features easily; therefore not yet optimized for the best performance. Ongoing work is towards large scale implementation and optimization of the software to show efficiency in order to advance the method to its fullest potential. Also, linking the error structure of the MMM method [3] to its adaptivity analysis in order to have a more mathematically sound adaptivity framework is left as a future work.

\section{Acknowledgements}

Financial support from the National Science Foundation (CMMI-1334763) is gratefully acknowledged. 


\section{References}

[1] Q. Yang, E. Biyikli, A.C. To, Multiresolution Molecular Mechanics: Statics, Computer Methods in Applied Mechanics and Engineering, (2013).

[2] E. Biyikli, Q. Yang, A.C. To, Multiresolution Molecular Mechanics: Dynamics, Computer Methods in Applied Mechanics and Engineering, 274 (2014) 42-55.

[3] Q. Yang, E. Biyikli, A.C. To, Multiresolution molecular mechanics: Convergence and error structure analysis, Computer Methods in Applied Mechanics and Engineering, 269 (2014) 20-45. [4] Q. Yang, A.C. To, Multiresolution molecular mechanics: A unified and consistent framework for general finite element shape functions, Computer Methods in Applied Mechanics and Engineering, (2014).

[5] P. Aubertin, J. Réthoré, R. De Borst, Energy conservation of atomistic/continuum coupling, International journal for numerical methods in engineering, 78 (2009) 1365-1386.

[6] M. Jebahi, F. Dau, J.-L. Charles, I. Iordanoff, Multiscale modeling of complex dynamic problems: an overview and recent developments, Archives of Computational Methods in Engineering, (2014) 1-38.

[7] F.F. Abraham, J.Q. Broughton, N. Bernstein, E. Kaxiras, Spanning the length scales in dynamic simulation, Computers in Physics, 12 (1998) 538-546.

[8] Y. Mo, K.T. Turner, I. Szlufarska, Friction laws at the nanoscale, Nature, 457 (2009) 11161119.

[9] R.E. Miller, E.B. Tadmor, The quasicontinuum method: Overview, applications and current directions, Journal of Computer-Aided Materials Design, 9 (2002) 203-239.

[10] R. Gracie, T. Belytschko, An adaptive concurrent multiscale method for the dynamic simulation of dislocations, International Journal for Numerical Methods in Engineering, 86 (2011) 575-597.

[11] F.F. Abraham, R. Walkup, H. Gao, M. Duchaineau, T.D. De La Rubia, M. Seager, Simulating materials failure by using up to one billion atoms and the world's fastest computer: Work-hardening, Proceedings of the National Academy of Sciences, 99 (2002) 5783-5787.

[12] J. Marian, J. Knap, G. Campbell, A quasicontinuum study of nanovoid collapse under uniaxial loading in Ta, Acta Materialia, 56 (2008) 2389-2399.

[13] R.E. Miller, E.B. Tadmor, Hybrid continuum mechanics and atomistic methods for simulating materials deformation and failure, MRS bulletin, 32 (2007) 920-926.

[14] E.B. Tadmor, M. Ortiz, R. Phillips, Quasicontinuum analysis of defects in solids, Philosophical Magazine A, 73 (1996) 1529-1563.

[15] B. Eidel, A. Stukowski, A variational formulation of the quasicontinuum method based on energy sampling in clusters, Journal of the Mechanics and Physics of Solids, 57 (2009) 87-108.

[16] J. Knap, M. Ortiz, An analysis of the quasicontinuum method, Journal of the Mechanics and Physics of Solids, 49 (2001) 1899-1923.

[17] D.M. Kochmann, G.N. Venturini, A meshless quasicontinuum method based on local maximum-entropy interpolation, Modelling and simulation in materials science and engineering, 22 (2014) 034007.

[18] T. Belytschko, S. Xiao, Coupling methods for continuum model with molecular model, International Journal for Multiscale Computational Engineering, 1 (2003).

[19] S. Xiao, T. Belytschko, A bridging domain method for coupling continua with molecular dynamics, Computer Methods in Applied Mechanics and Engineering, 193 (2004) 1645-1669. 
[20] M. Xu, T. Belytschko, Conservation properties of the bridging domain method for coupled molecular/continuum dynamics, International Journal for Numerical Methods in Engineering, 76 (2008) 278-294.

[21] G.J. Wagner, W.K. Liu, Coupling of atomistic and continuum simulations using a bridging scale decomposition, Journal of Computational Physics, 190 (2003) 249-274.

[22] D. Qian, G.J. Wagner, W.K. Liu, A multiscale projection method for the analysis of carbon nanotubes, Computer Methods in Applied Mechanics and Engineering, 193 (2004) 1603-1632.

[23] H.S. Park, E.G. Karpov, W.K. Liu†, P.A. Klein, The bridging scale for two-dimensional atomistic/continuum coupling, Philosophical Magazine, 85 (2005) 79-113.

[24] L. Shilkrot, R. Miller, W. Curtin, Coupled atomistic and discrete dislocation plasticity, Physical Review Letters, 89 (2002) 025501.

[25] S. Qu, V. Shastry, W. Curtin, R. Miller, A finite-temperature dynamic coupled atomistic/discrete dislocation method, Modelling and simulation in materials science and engineering, 13 (2005) 1101.

[26] L. Xiong, Q. Deng, G. Tucker, D.L. McDowell, Y. Chen, A concurrent scheme for passing dislocations from atomistic to continuum domains, Acta Materialia, 60 (2012) 899-913.

[27] L. Xiong, Q. Deng, G.J. Tucker, D.L. McDowell, Y. Chen, Coarse-grained atomistic simulations of dislocations in $\mathrm{Al}, \mathrm{Ni}$ and $\mathrm{Cu}$ crystals, International Journal of Plasticity, 38 (2012) 86-101.

[28] S. Badia, P. Bochev, J. Fish, M. Gunzburger, R. Lehoucq, M. Nuggehally, M. Parks, A force-based blending model for atomistic-to-continuum coupling, International Journal for Multiscale Computational Engineering, 5 (2007) 387-406.

[29] J. Fish, M.A. Nuggehally, M.S. Shephard, C.R. Picu, S. Badia, M.L. Parks, M. Gunzburger, Concurrent $\mathrm{AtC}$ coupling based on a blend of the continuum stress and the atomistic force, Computer Methods in Applied Mechanics and Engineering, 196 (2007) 4548-4560.

[30] Q. Yang, E. Biyikli, P. Zhang, R. Tian, A.C. To, Atom collocation method, Computer Methods in Applied Mechanics and Engineering, 237 (2012) 67-77.

[31] H.S. Park, P.A. Klein, G.J. Wagner, A surface Cauchy-Born model for nanoscale materials, International Journal for Numerical Methods in Engineering, 68 (2006) 1072-1095.

[32] A.C. To, S. Li, Perfectly matched multiscale simulations, Physical Review B, 72 (2005) 035414.

[33] N.M. Ghoniem†े, E.P. Busso, N. Kioussis, H. Huang, Multiscale modelling of nanomechanics and micromechanics: an overview, Philosophical magazine, 83 (2003) 34753528.

[34] R.E. Miller, E. Tadmor, A unified framework and performance benchmark of fourteen multiscale atomistic/continuum coupling methods, Modelling and simulation in materials science and engineering, 17 (2009) 053001.

[35] J.Q. Broughton, F.F. Abraham, N. Bernstein, E. Kaxiras, Concurrent coupling of length scales: methodology and application, Physical review B, 60 (1999) 2391.

[36] W.A. Curtin, R.E. Miller, Atomistic/continuum coupling in computational materials science, Modelling and simulation in materials science and engineering, 11 (2003) R33.

[37] J. Fish, Bridging the scales in nano engineering and science, Journal of Nanoparticle Research, 8 (2006) 577-594.

[38] W.K. Liu, E. Karpov, S. Zhang, H. Park, An introduction to computational nanomechanics and materials, Computer Methods in Applied Mechanics and Engineering, 193 (2004) 15291578. 
[39] G. Lu, E. Kaxiras, An overview of multiscale simulations of materials, arXiv preprint condmat/0401073, (2004).

[40] H.S. Park, W.K. Liu, An introduction and tutorial on multiple-scale analysis in solids, Computer Methods in Applied Mechanics and Engineering, 193 (2004) 1733-1772.

[41] J. Wernik, S.A. Meguid, Coupling atomistics and continuum in solids: status, prospects, and challenges, International Journal of Mechanics and Materials in Design, 5 (2009) 79-110.

[42] V. Shenoy, R. Miller, E. Tadmor, D. Rodney, R. Phillips, M. Ortiz, An adaptive finite element approach to atomic-scale mechanics - the quasicontinuum method, Journal of the Mechanics and Physics of Solids, 47 (1999) 611-642.

[43] J.Y. Park, S. Im, Adaptive nonlocal quasicontinuum for deformations of curved crystalline structures, Physical Review B, 77 (2008) 184109.

[44] S. Kwon, Y. Lee, J.Y. Park, D. Sohn, J.H. Lim, S. Im, An efficient three-dimensional adaptive quasicontinuum method using variable-node elements, Journal of Computational Physics, 228 (2009) 4789-4810.

[45] T. Shimokawa, T. Kinari, S. Shintaku, Adaptive Mesh Refinement with Elastic Stiffness Coefficients in the Quasicontinuum Model, Journal of Computational Science and Technology, 3 (2009) 408-416.

[46] W. Shan, U. Nackenhorst, An adaptive FE-MD model coupling approach, Computational Mechanics, 46 (2010) 577-596.

[47] P. Moseley, J. Oswald, T. Belytschko, Adaptive atomistic-to-continuum modeling of propagating defects, International Journal for Numerical Methods in Engineering, 92 (2012) 835856.

[48] P. Moseley, J. Oswald, T. Belytschko, ADAPTIVE ATOMISTIC-CONTINUUM MODELING OF DEFECT INTERACTION WITH THE DEBDM, International Journal for Multiscale Computational Engineering, 11 (2013).

[49] E. Marenic, J. Soric, A. Ibrahimbegovic, Adaptive modelling in atomistic-to-continuum multiscale methods, Journal of the Serbian Society for Computational Mechanics/Vol, 6 (2012) 169-198.

[50] M. Praprotnik, L. Delle Site, K. Kremer, Adaptive resolution molecular-dynamics simulation: Changing the degrees of freedom on the fly, The Journal of chemical physics, 123 (2005) 224106.

[51] A. Heyden, D.G. Truhlar, Conservative algorithm for an adaptive change of resolution in mixed atomistic/coarse-grained multiscale simulations, Journal of Chemical Theory and Computation, 4 (2008) 217-221.

[52] J. Tinsley Oden, S. Prudhomme, A. Romkes, P.T. Bauman, Multiscale modeling of physical phenomena: Adaptive control of models, SIAM Journal on Scientific Computing, 28 (2006) 2359-2389.

[53] P.R. Budarapu, R. Gracie, S.P. Bordas, T. Rabczuk, An adaptive multiscale method for quasi-static crack growth, Computational Mechanics, 53 (2014) 1129-1148.

[54] S.-W. Yang, P.R. Budarapu, D.R. Mahapatra, S.P. Bordas, G. Zi, T. Rabczuk, A meshless adaptive multiscale method for fracture, Computational Materials Science, 96 (2015) 382-395.

[55] R. Tong, G. Liu, L. Liu, L. Wu, Adaptive multiscale method for two-dimensional nanoscale adhesive contacts, Chinese Journal of Mechanical Engineering, 26 (2013) 606-612.

[56] W. Hankui, Z. Xiong, Q. Xinming, Adaptive smoothed molecular dynamics for multiscale modeling, Computational Materials Science, 46 (2009) 713-715. 
[57] R. Miller, E. Tadmor, R. Phillips, M. Ortiz, Quasicontinuum simulation of fracture at the atomic scale, Modelling and simulation in materials science and engineering, 6 (1998) 607.

[58] H.J. Berendsen, J.P.M. Postma, W.F. van Gunsteren, A. DiNola, J. Haak, Molecular dynamics with coupling to an external bath, The Journal of chemical physics, 81 (1984) 36843690 .

[59] L. Kalé, R. Skeel, M. Bhandarkar, R. Brunner, A. Gursoy, N. Krawetz, J. Phillips, A. Shinozaki, K. Varadarajan, K. Schulten, NAMD2: greater scalability for parallel molecular dynamics, Journal of Computational Physics, 151 (1999) 283-312.

[60] D.A. Pearlman, D.A. Case, J.W. Caldwell, W.S. Ross, T.E. Cheatham, S. DeBolt, D. Ferguson, G. Seibel, P. Kollman, AMBER, a package of computer programs for applying molecular mechanics, normal mode analysis, molecular dynamics and free energy calculations to simulate the structural and energetic properties of molecules, Computer Physics Communications, 91 (1995) 1-41.

[61] B. Luan, S. Hyun, J. Molinari, N. Bernstein, M.O. Robbins, Multiscale modeling of twodimensional contacts, Physical Review E, 74 (2006) 046710.

[62] S. Plimpton, Fast parallel algorithms for short-range molecular dynamics, Journal of Computational Physics, 117 (1995) 1-19.

[63] A. Stukowski, Visualization and analysis of atomistic simulation data with OVITO-the Open Visualization Tool, Modelling and Simulation in Materials Science and Engineering, 18 (2010) 015012.

[64] D. Hull, D.J. Bacon, Introduction to dislocations, Pergamon Press Oxford, 1984.

[65] C.L. Kelchner, S. Plimpton, J. Hamilton, Dislocation nucleation and defect structure during surface indentation, Physical Review B, 58 (1998) 11085.

\section{Figure captions}

Figure 1: Interesting atoms take only $8 \%$ of the entire system in a nanoindentation example.

Figure 2: Types of atoms: Interpolating rep-atom (red), non-interpolating rep-atom (black), primary sampling atom (blue), secondary sampling atom (green), non-sampling atom (gray).

Figure 3: A Gaussian wave is introduced in the center and atoms are fixed at the ends in the 1-D wave propagation example.

Figure 4: Part of the 1-D wave model includes right half of the wave in full atomistic region and an element in the coarsened region.

Figure 5: Comparison of trajectories of full atomistic and MMD model at the beginning (a, b), intermediate $(c, d)$ and end $(e, f)$ of the 1-D wave propagation. 
Figure 6: Kinetic energy of the element in the red box is monitored in the 1-D wave propagation example.

Figure 7: Comparison of monitored kinetic energies of full atomistic and MMD in the 1-D wave propagation example along with the relative difference (i.e., $\mid$ Full Atomistic - MMD / Full Atomistic.

Figure 8: 2-D dislocation example setup (left): Bottom is fixed and top is pulled; and, MMD model atom types (right).

Figure 9: Comparison of trajectories of full-atomistic and MMD model at the beginning (a), intermediate (b) and end (c) of the 2-D dislocation example. Coloring scheme of different atom types: Interpolating rep-atom (red), non-interpolating rep-atom (black), primary sampling atom (blue), secondary sampling atom (green), and non-sampling atom (gray).

Figure 10: Comparison of force versus strain curves of full atomistic and MMD in the 2-D dislocation example.

Figure 11: 3-D nanoindentation example setup (left): Bottom and lateral are fixed and top is indented; and, MMD model atom types (right).

Figure 12: Comparison of configurations at the onset of plastic deformation in the 3-D nanoindentation example where atoms are colored with respect to their potential energies: Full atomistic (a), MMD with criterion $-2.47 \mathrm{eV}$ (b), and MMD with criterion $-2.50 \mathrm{eV}$ (c).

Figure 13: Comparison of configurations at a later stage of plastic deformation in the 3-D nanoindentation example where blue atoms denote dislocation and red atoms denote stacking faults: Full atomistic (a), MMD with criterion $-2.47 \mathrm{eV}(\mathrm{b})$, and MMD with criterion $-2.50 \mathrm{eV}$ (c).

Figure 14: Comparison of centrosymmetry parameter histogram of full atomistic and MMD models with criterion $-2.47 \mathrm{eV}$ and $-2.50 \mathrm{eV}$ in the 3-D nanoindentation example.

Figure 15: Comparison of force versus depth curves of full atomistic and MMD models with criterion $-2.47 \mathrm{eV}$ (left) and $-2.50 \mathrm{eV}$ (right) in the 3-D nanoindentation example. 
Figure 16: Comparison of atom types of MMD models with criterion $-2.47 \mathrm{eV}$ (left) and -2.50 $\mathrm{eV}$ (right) in the 3-D nanoindentation example. 


\section{Tables}

Table 1: Descriptions of 5 types of atoms employed in MMM.

\begin{tabular}{|c|c|c|c|c|}
\hline $\begin{array}{c}\text { General } \\
\text { name }\end{array}$ & $\begin{array}{l}\text { Specific } \\
\text { name }\end{array}$ & $\begin{array}{l}\text { Degree of } \\
\text { freedom }\end{array}$ & Energy & Description \\
\hline \multirow[t]{2}{*}{ Rep-atom } & $\begin{array}{l}\text { Interpolating } \\
\text { rep-atom }\end{array}$ & Representative & Individual & $\begin{array}{l}\text { Nodes of the mesh, } \\
\text { interpolates for the } \\
\text { positions of ghost } \\
\text { atoms }\end{array}$ \\
\hline & $\begin{array}{c}\text { Non-interpolating } \\
\text { rep-atom }\end{array}$ & Individual & Individual & $\begin{array}{l}\text { Same as atoms of full } \\
\text { atomistic }\end{array}$ \\
\hline \multirow{3}{*}{ Ghost-atom } & $\begin{array}{c}\text { Primary } \\
\text { sampling atom }\end{array}$ & Passive & Representative & $\begin{array}{c}\text { Samples for the } \\
\text { energy of } \\
\text { non-sampling atoms }\end{array}$ \\
\hline & $\begin{array}{c}\text { Secondary } \\
\text { sampling atom }\end{array}$ & Passive & Individual & $\begin{array}{l}\text { Samples its own } \\
\text { energy }\end{array}$ \\
\hline & $\begin{array}{c}\text { Non-sampling } \\
\text { atom }\end{array}$ & Passive & Passive & $\begin{array}{l}\text { Contributes to } \\
\text { atomistic description }\end{array}$ \\
\hline
\end{tabular}


Table 2: Refinement procedure of MMM adaptivity.

\section{Refinement Procedure}

1. Return if it is not time to query adaptivity.

2. Activate ghost atoms with potential energies higher than the threshold value, return if none.

3. Activate atoms within a radius of influence of the activated atoms.

4. Activate atoms inside elements that include activated atoms.

5. Convert activated atom types to non-interpolating rep-atoms.

6. Interpolate new velocities of activated atoms from corresponding interpolating repatoms.

7. Default masses of activated atoms and reduce masses of corresponding interpolating repatoms accordingly.

8. Recalculate energies and forces of all atoms for the current iteration.

Table 3: Coarsening procedure of MMM adaptivity.

\section{Coarsening Procedure}

1. Return if it is not time to query adaptivity.

2. Select rep-atoms with potential energies lower than the threshold value, return if none.

3. Activate elements full of the selected atoms.

4. Activate all atoms inside activated elements.

5. Convert activated atom types to scheme types.

6. Extrapolate velocities and masses of activated atoms to corresponding interpolating repatoms.

7. Recalculate energies and forces of all atoms for the current iteration. 


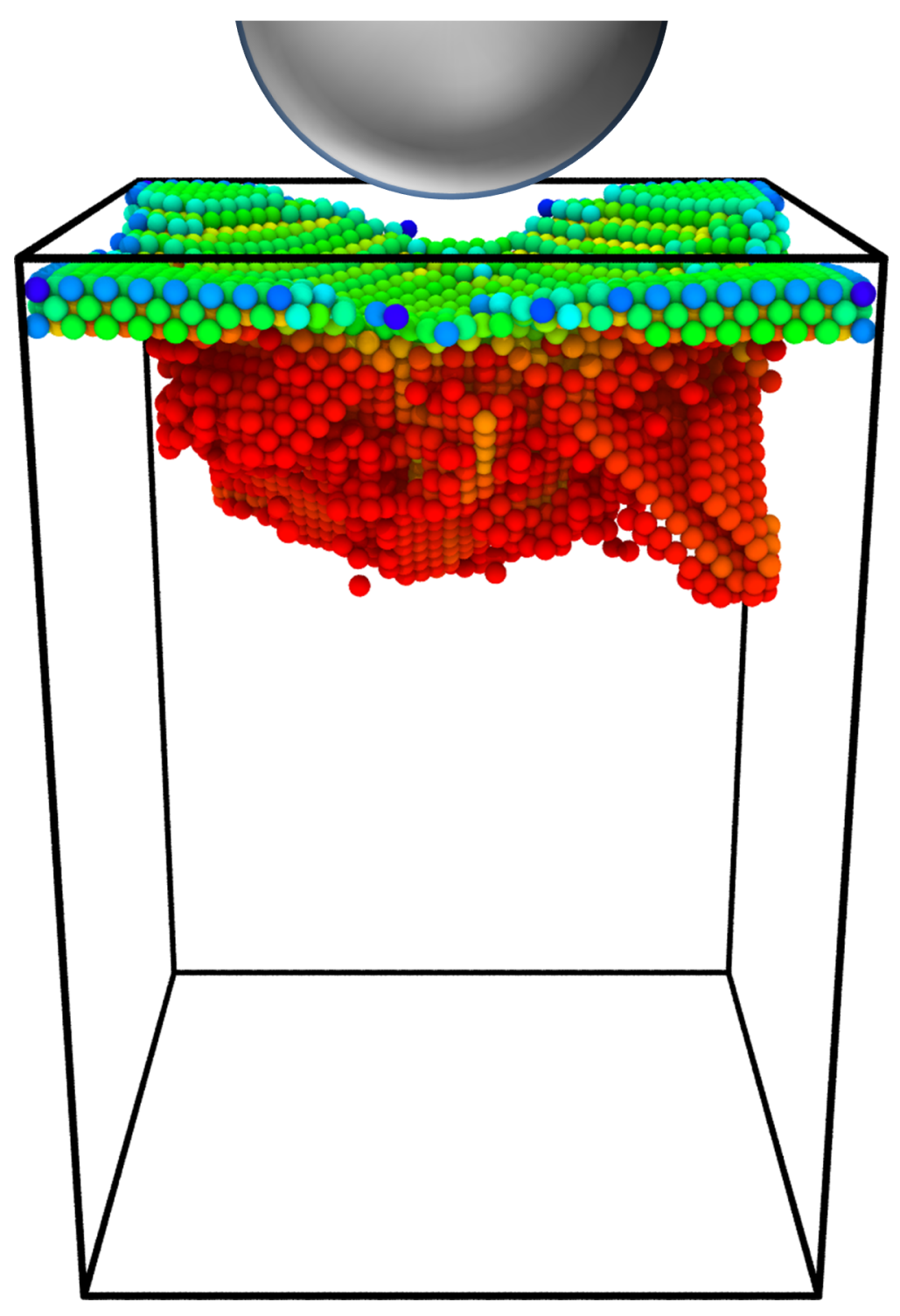


Figure 2

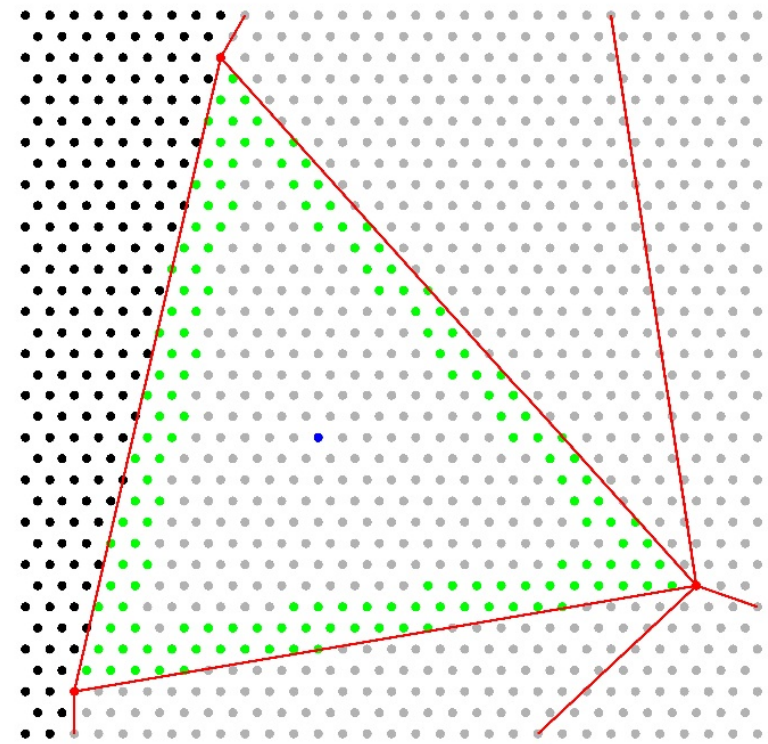


Figure 3

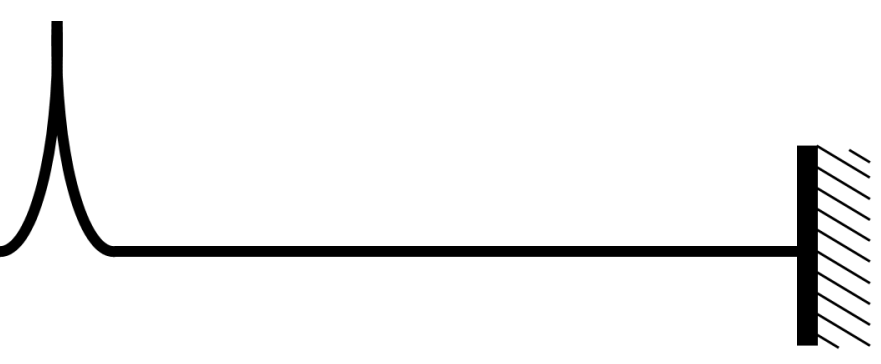




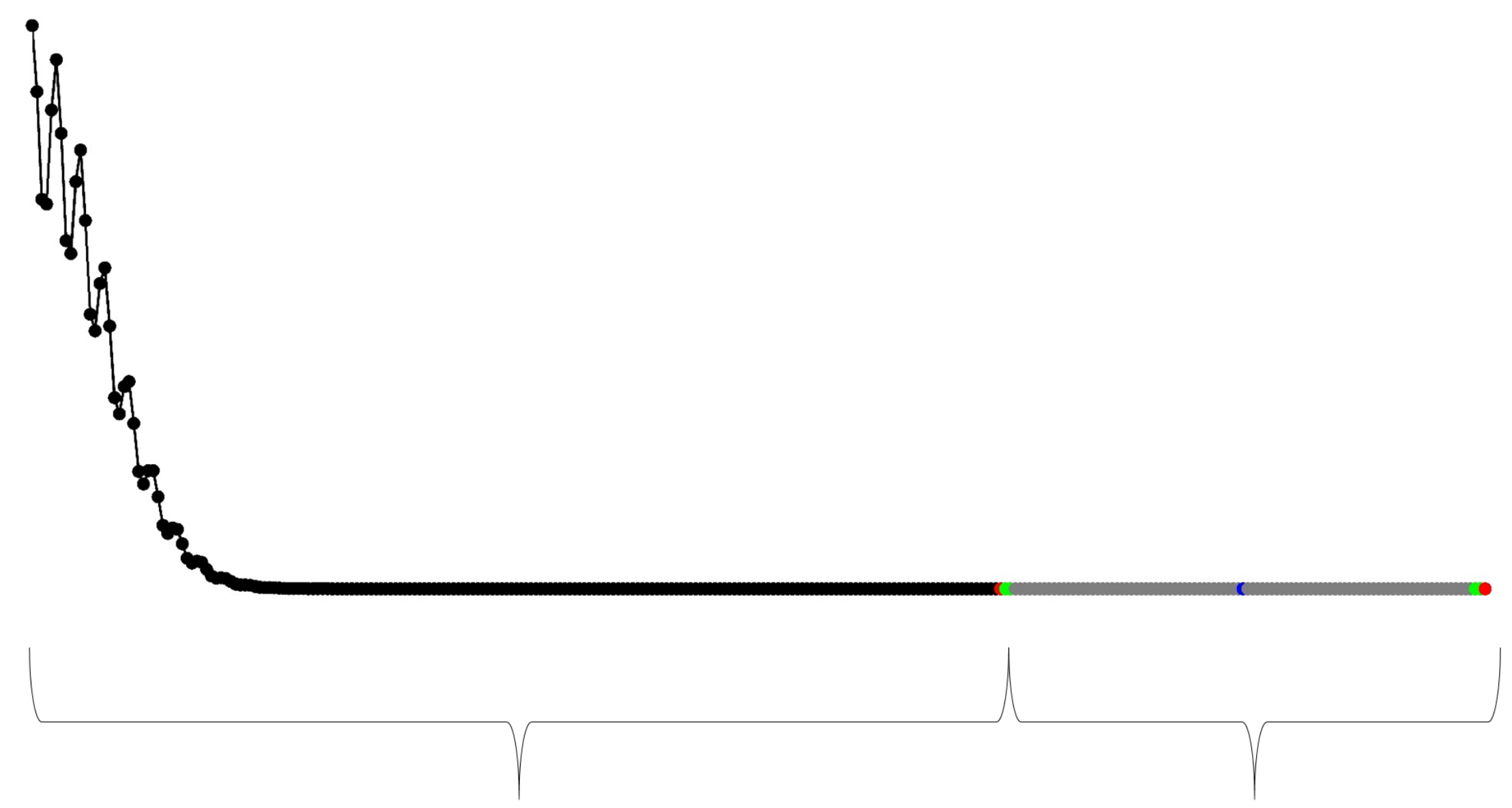

Fine region

\section{Coarse region}


(a)

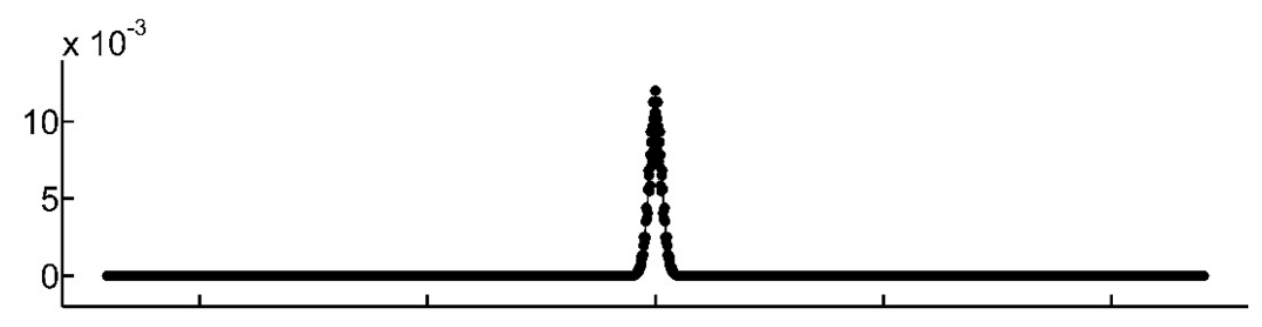

(b)

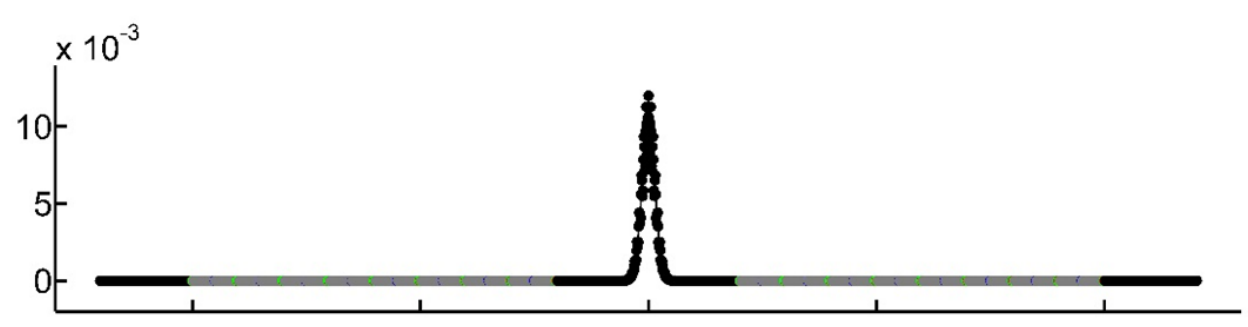

(c)

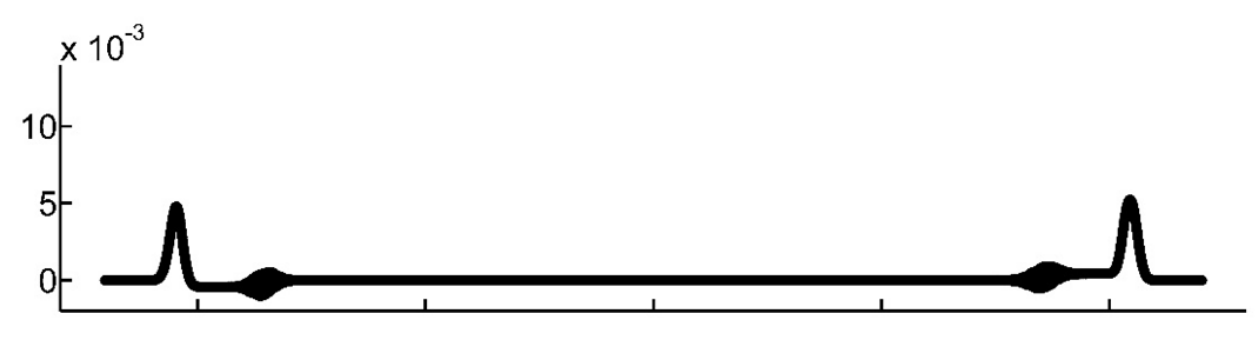

(e)

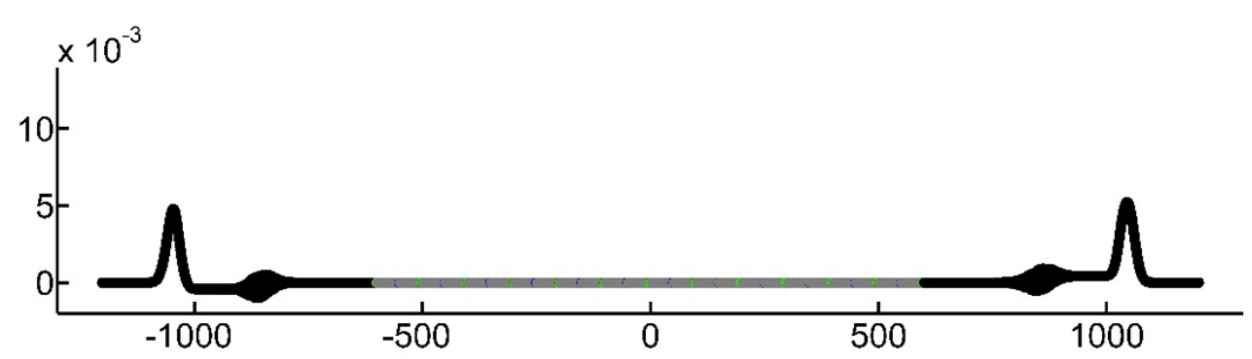

Position ( $\AA$ ) 


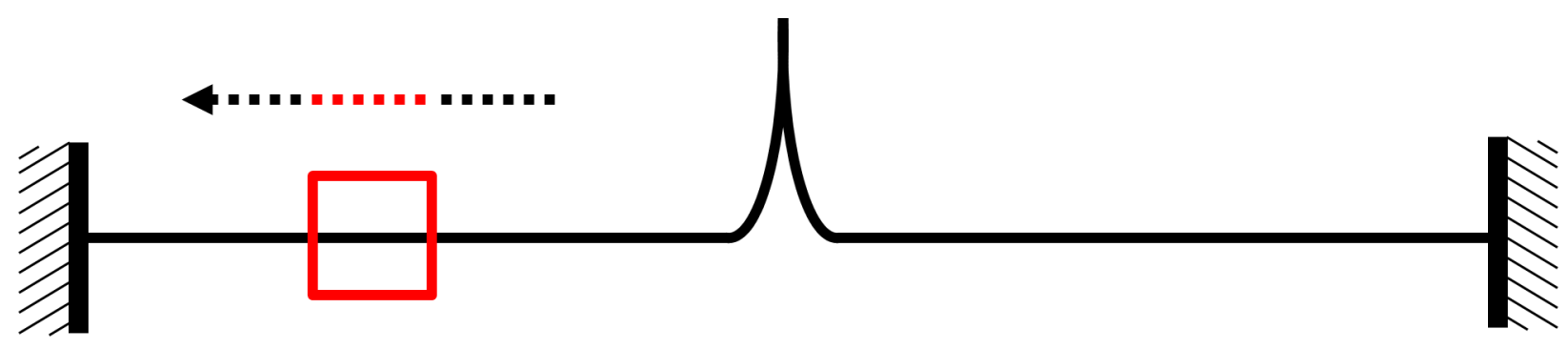




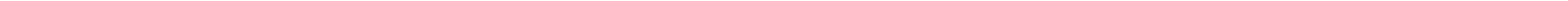


Figure 8
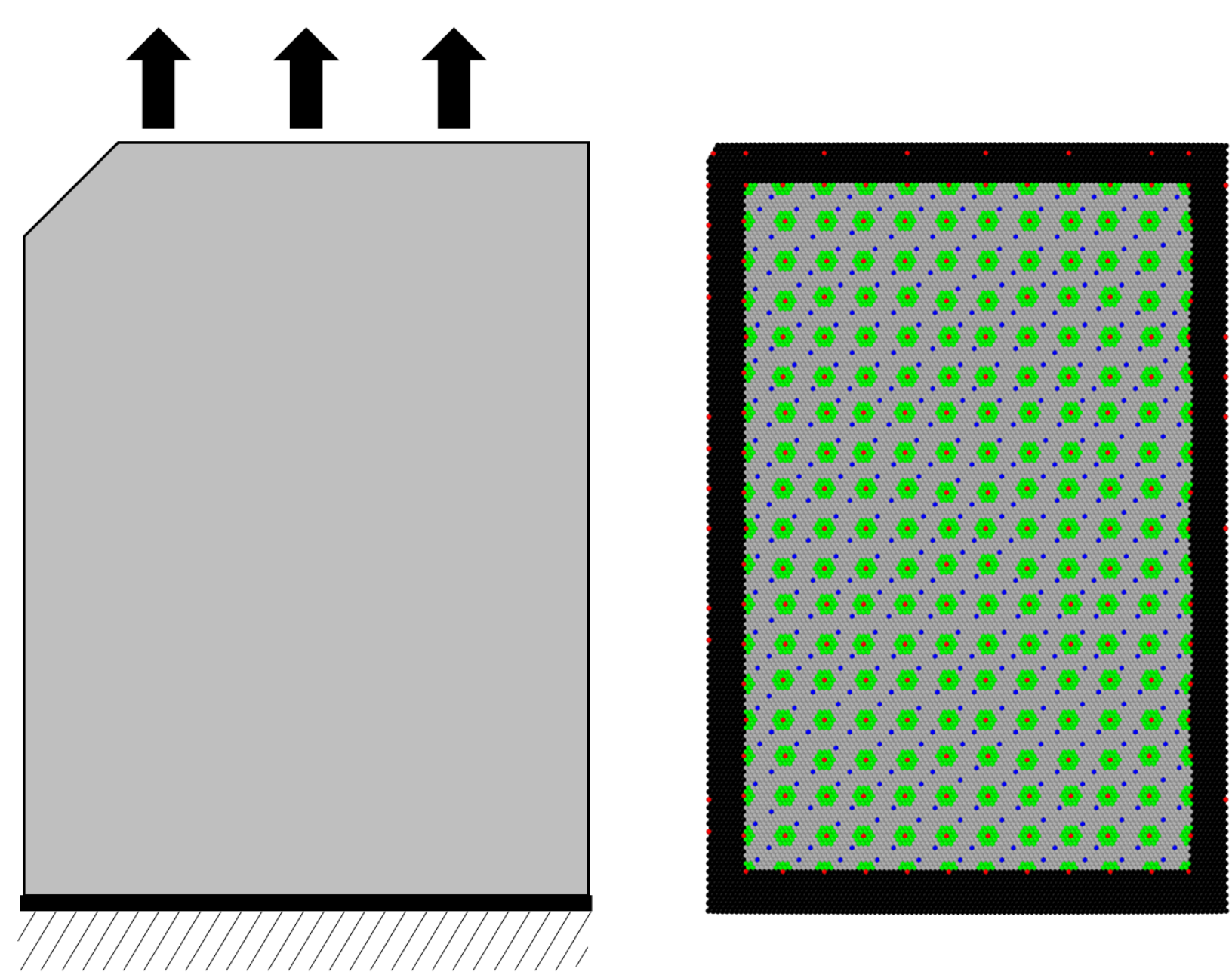


\section{Figure 9}

(a)
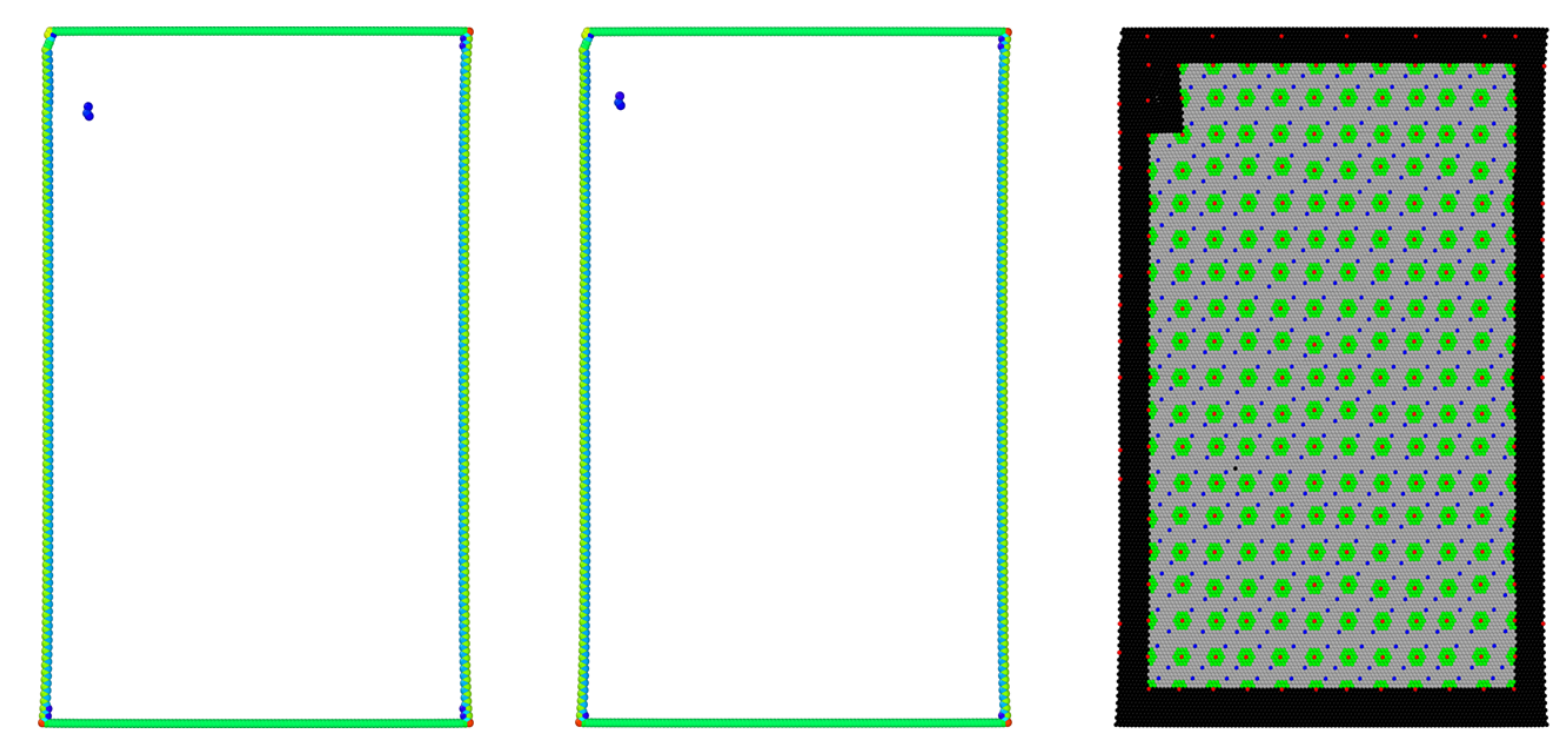

(b)
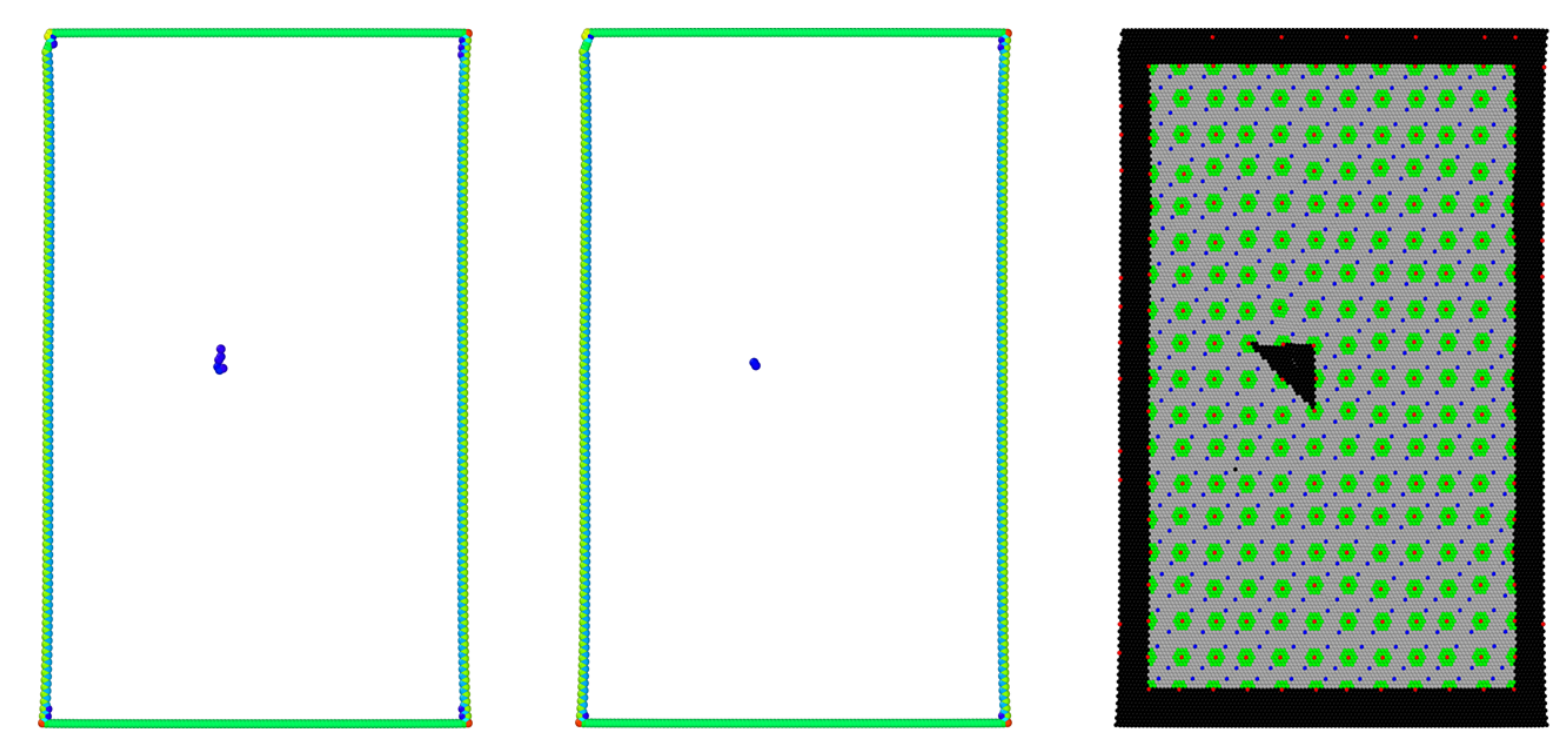

(c)
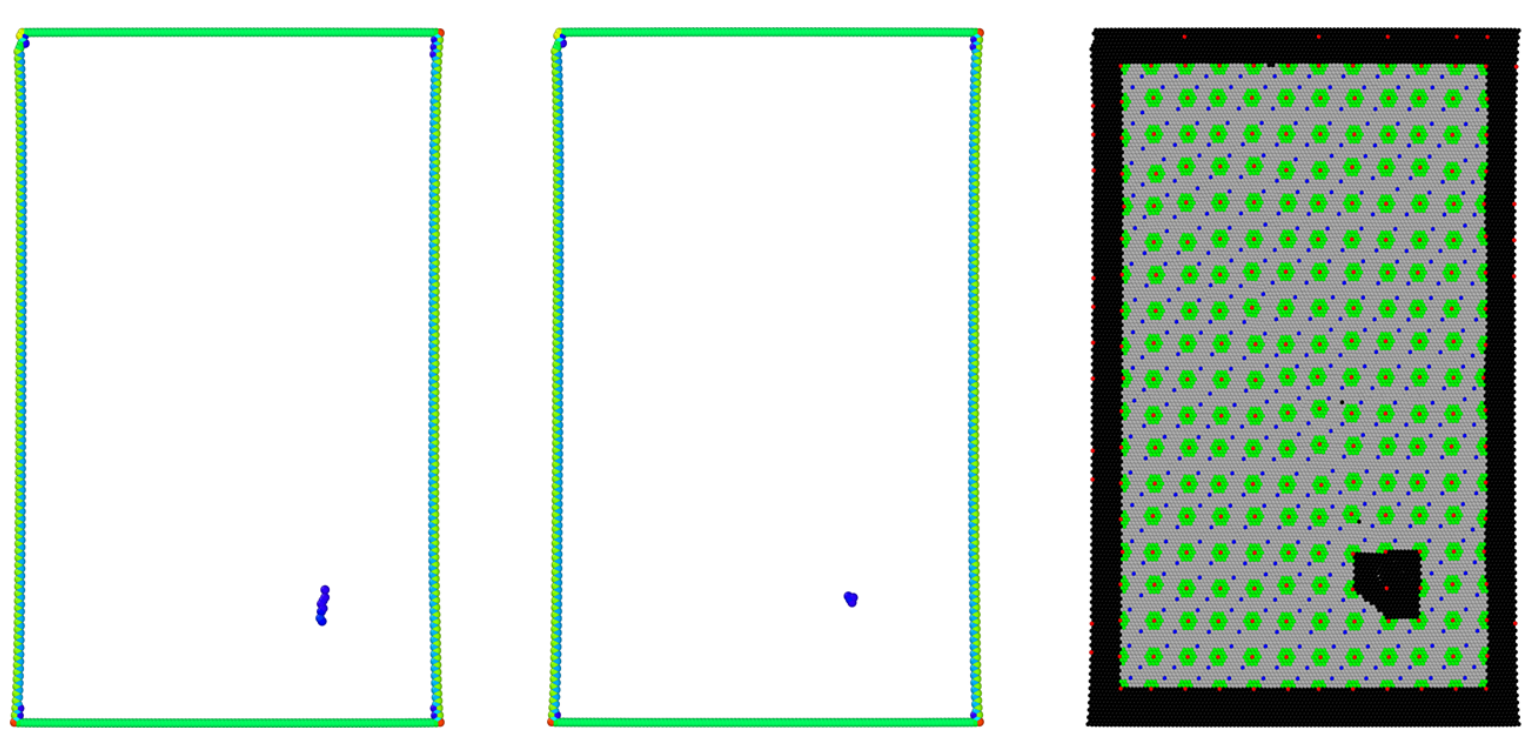

Full Atomistic MMD 
Figure 10

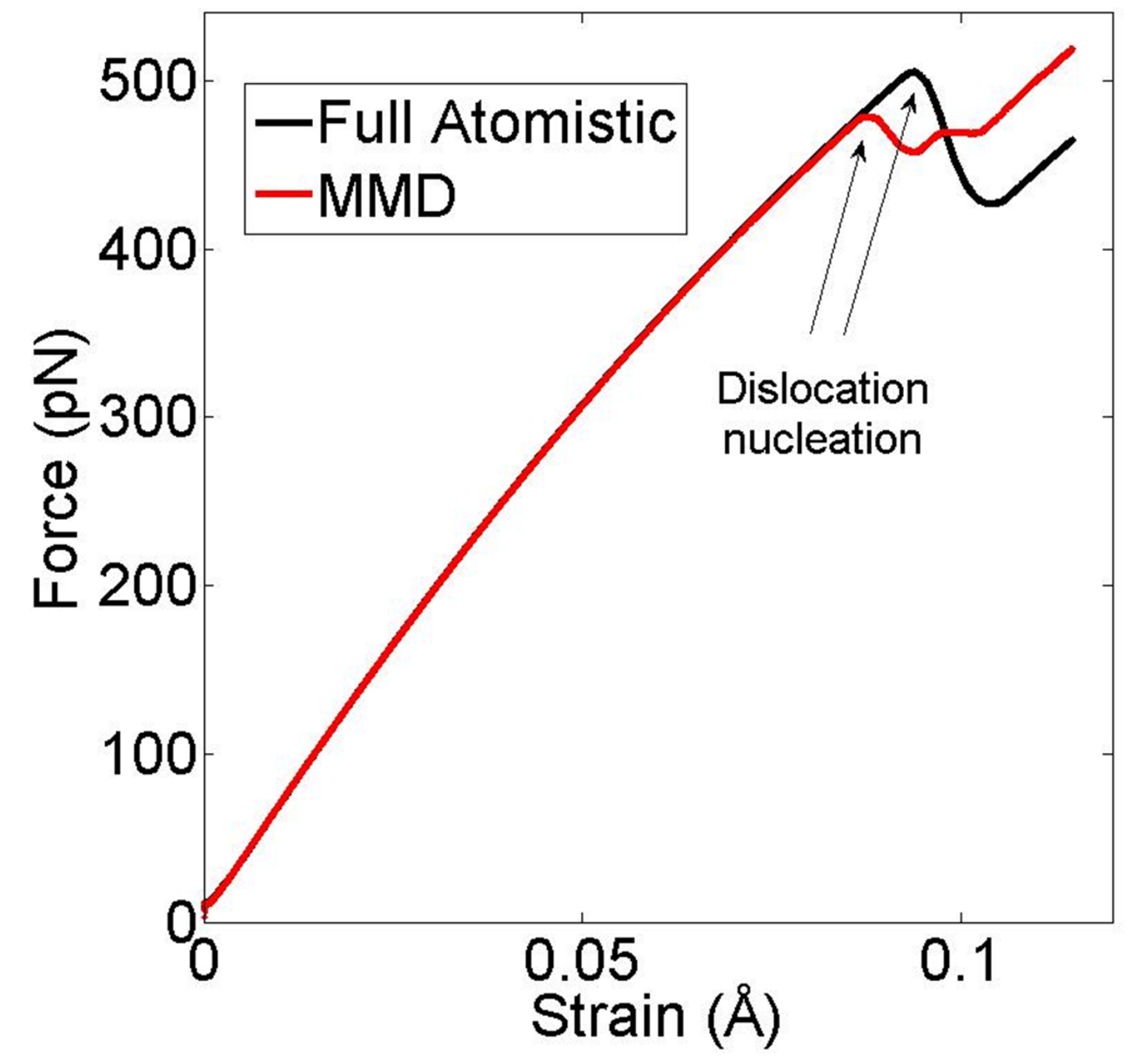


Figure 11
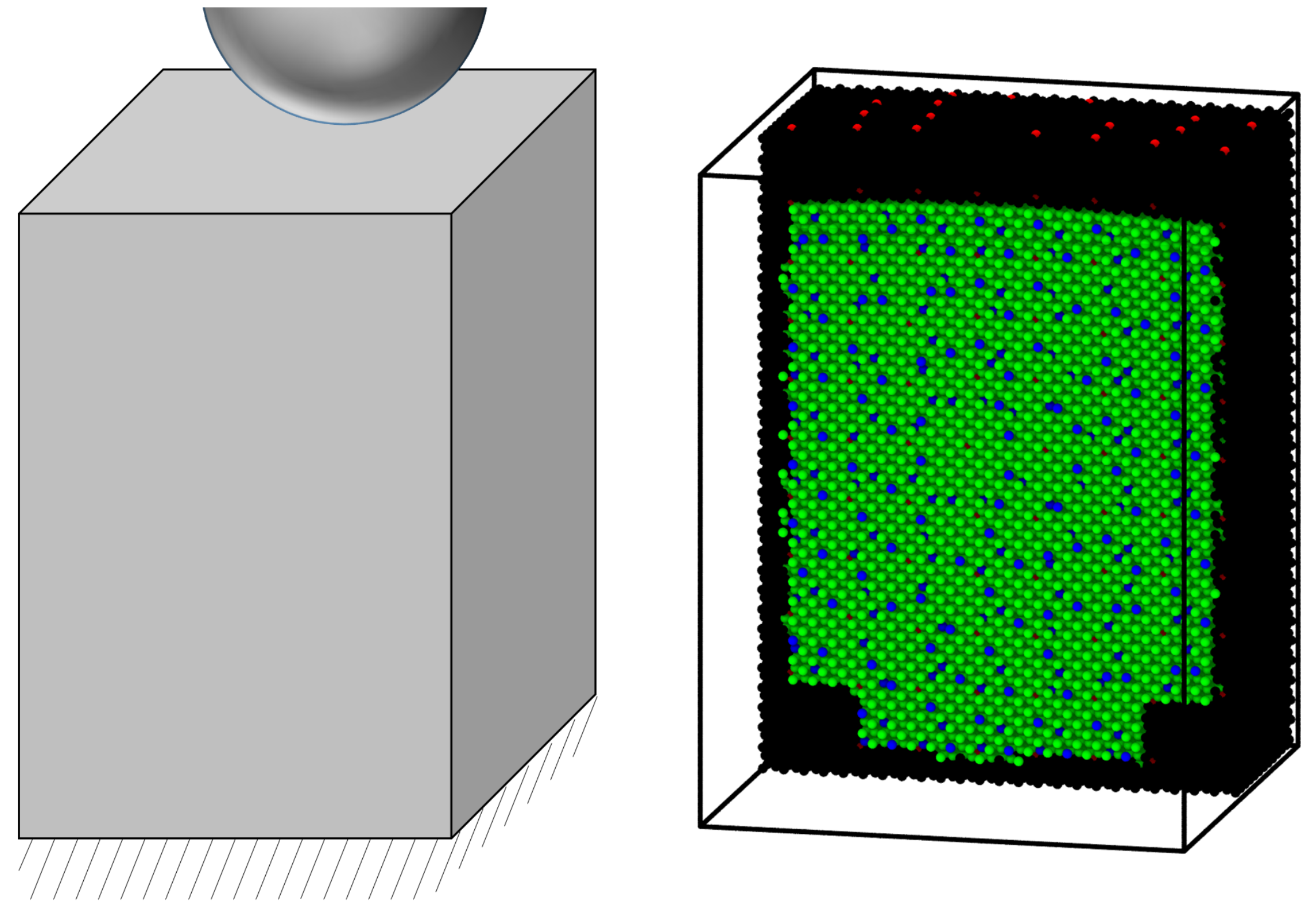
Figure 12

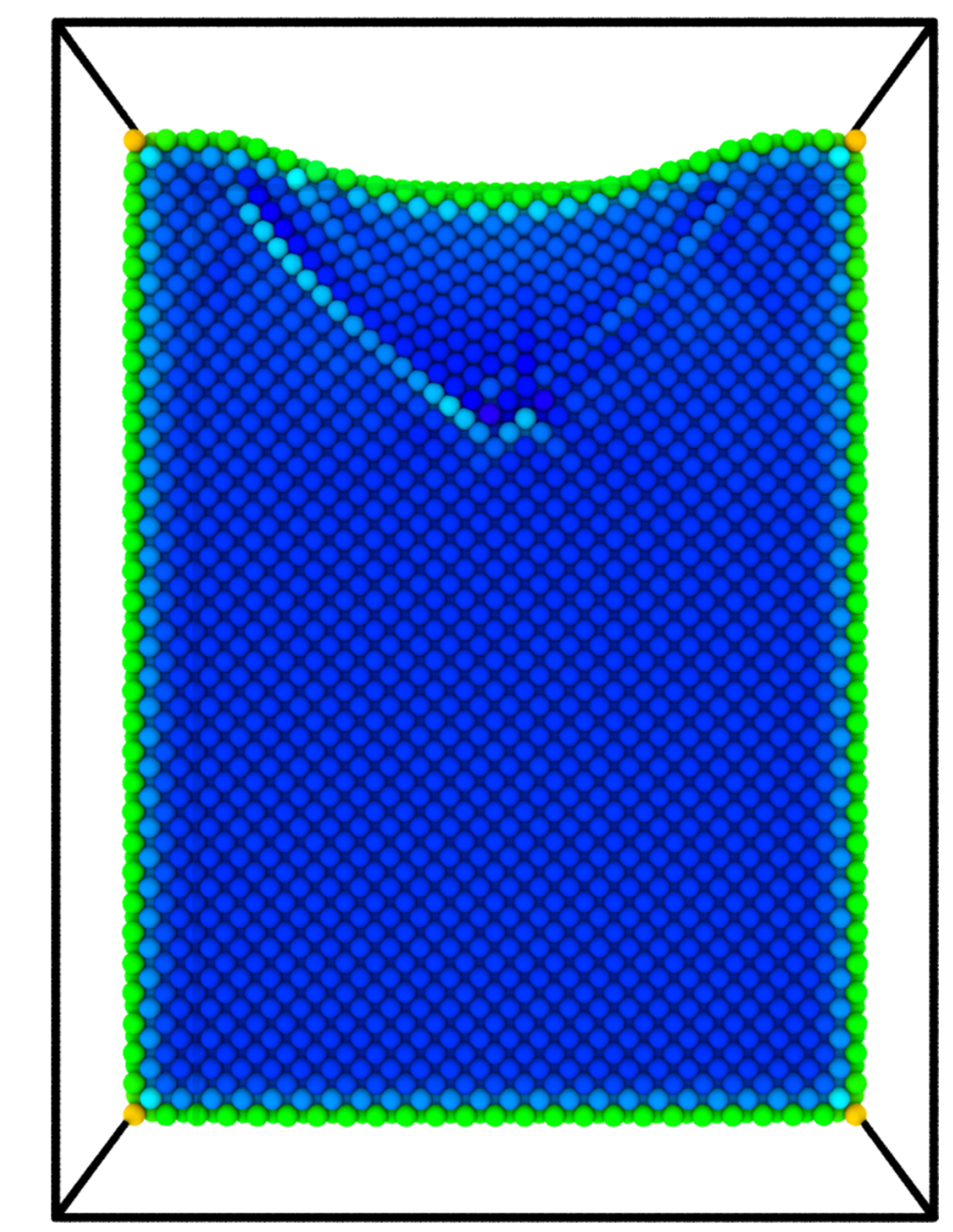

(a)

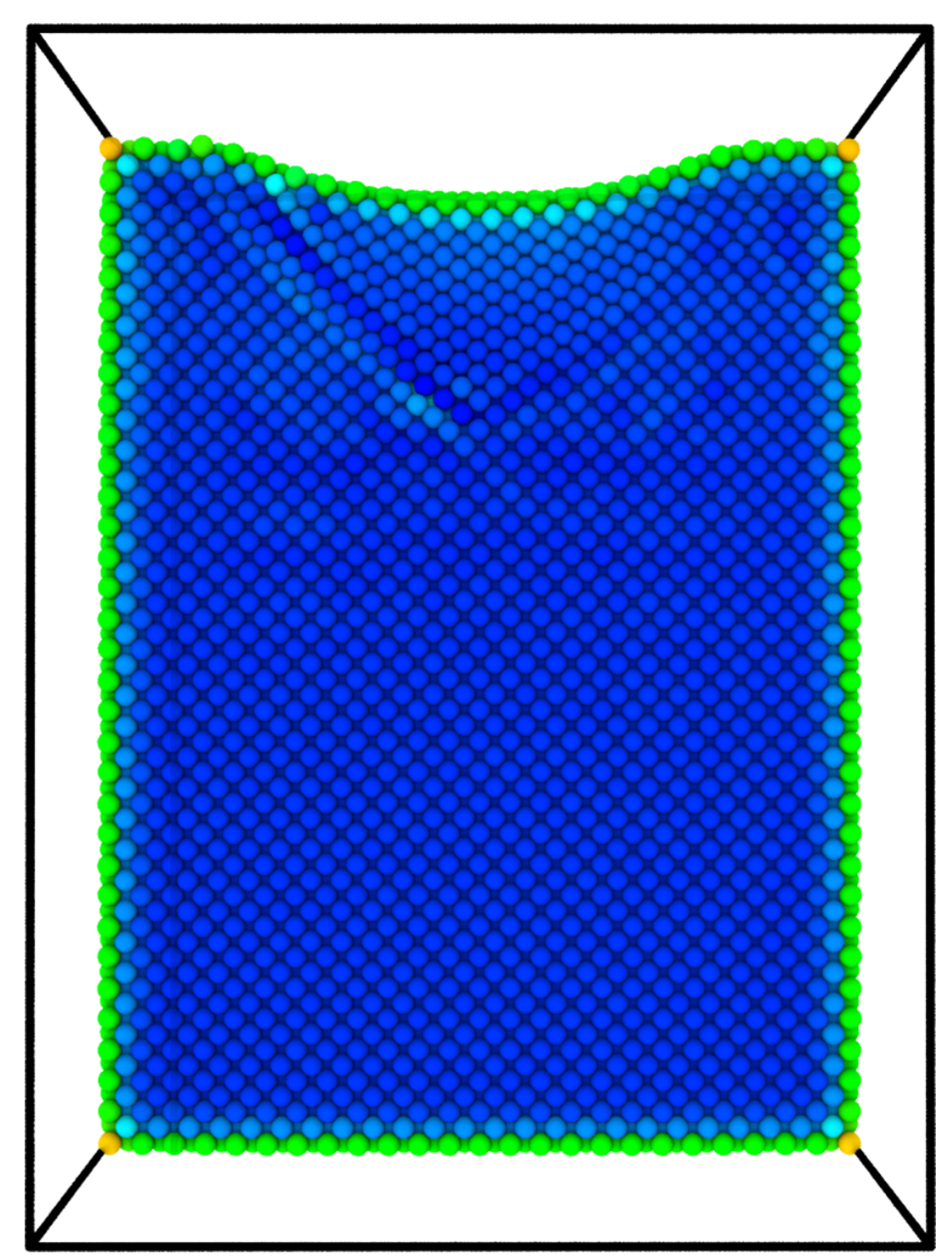

(b)

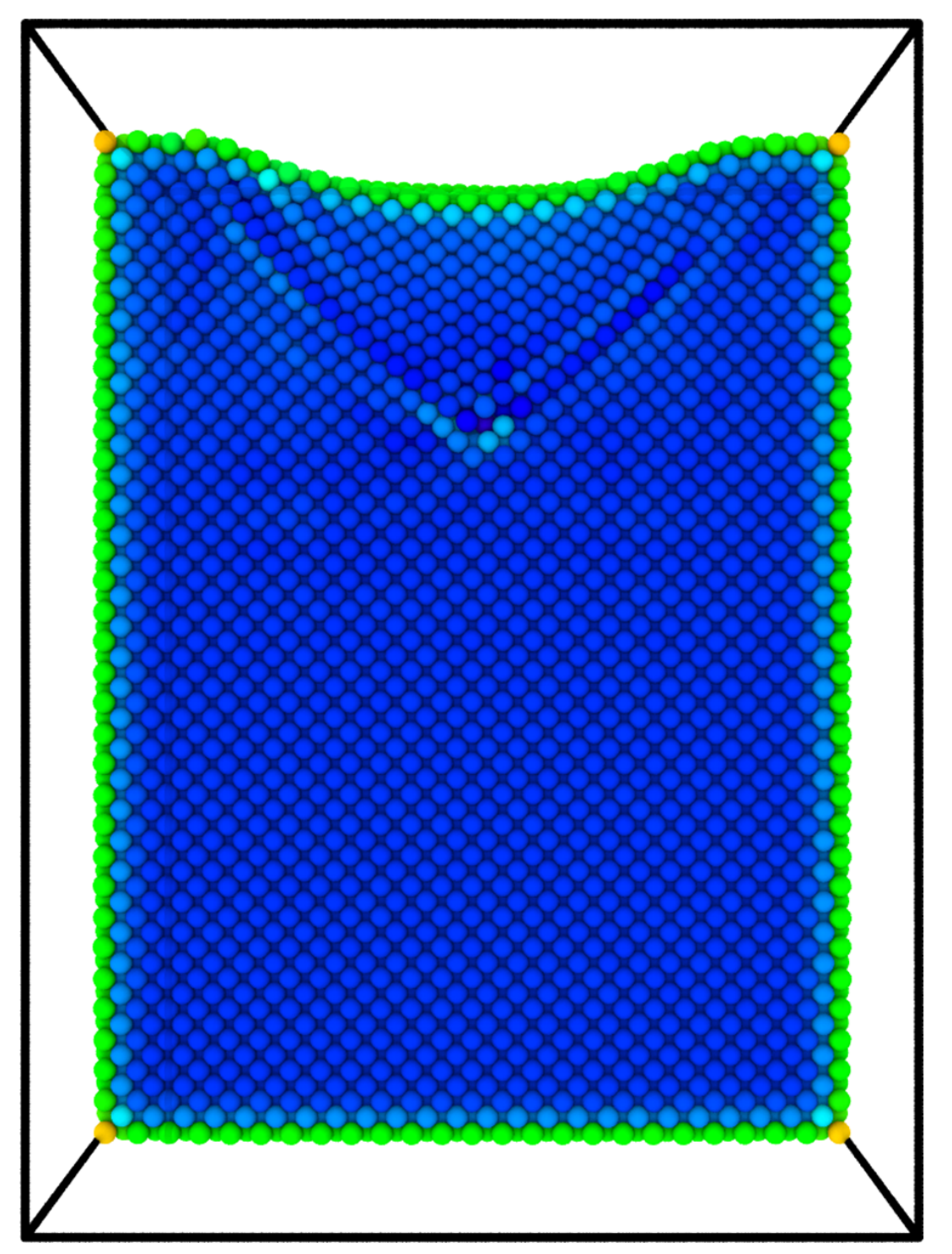

(c) 
Figure 13

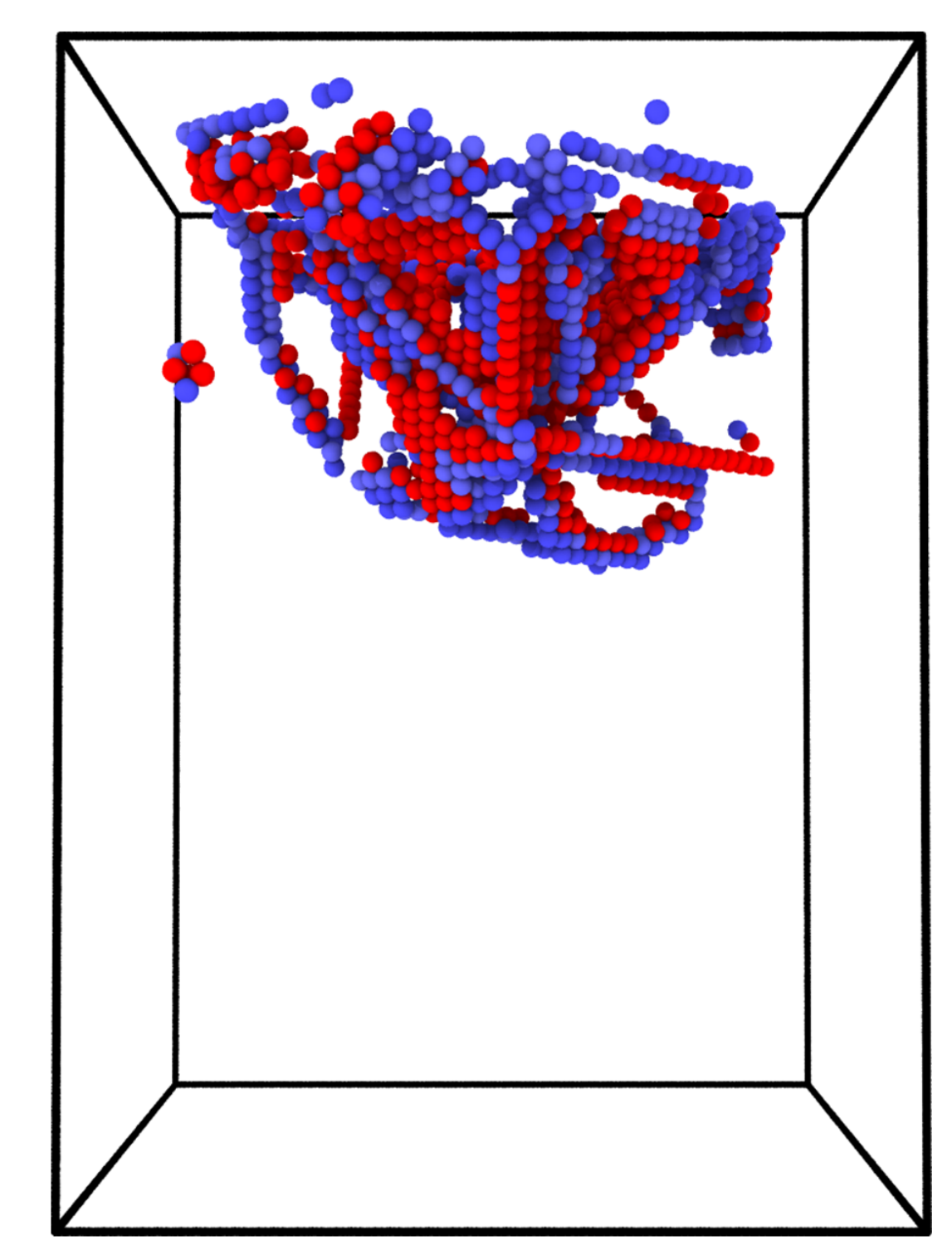

(a)

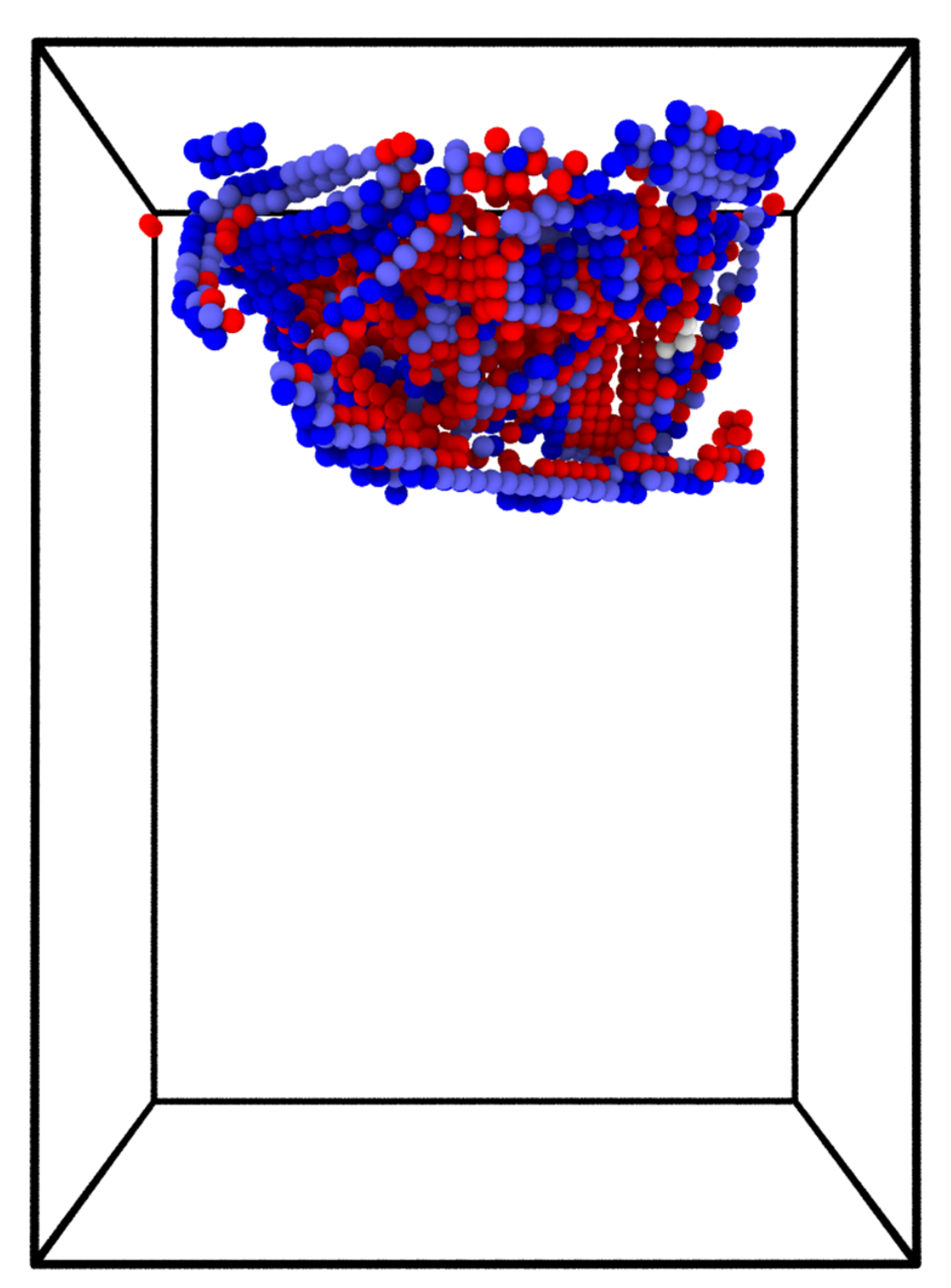

(b)

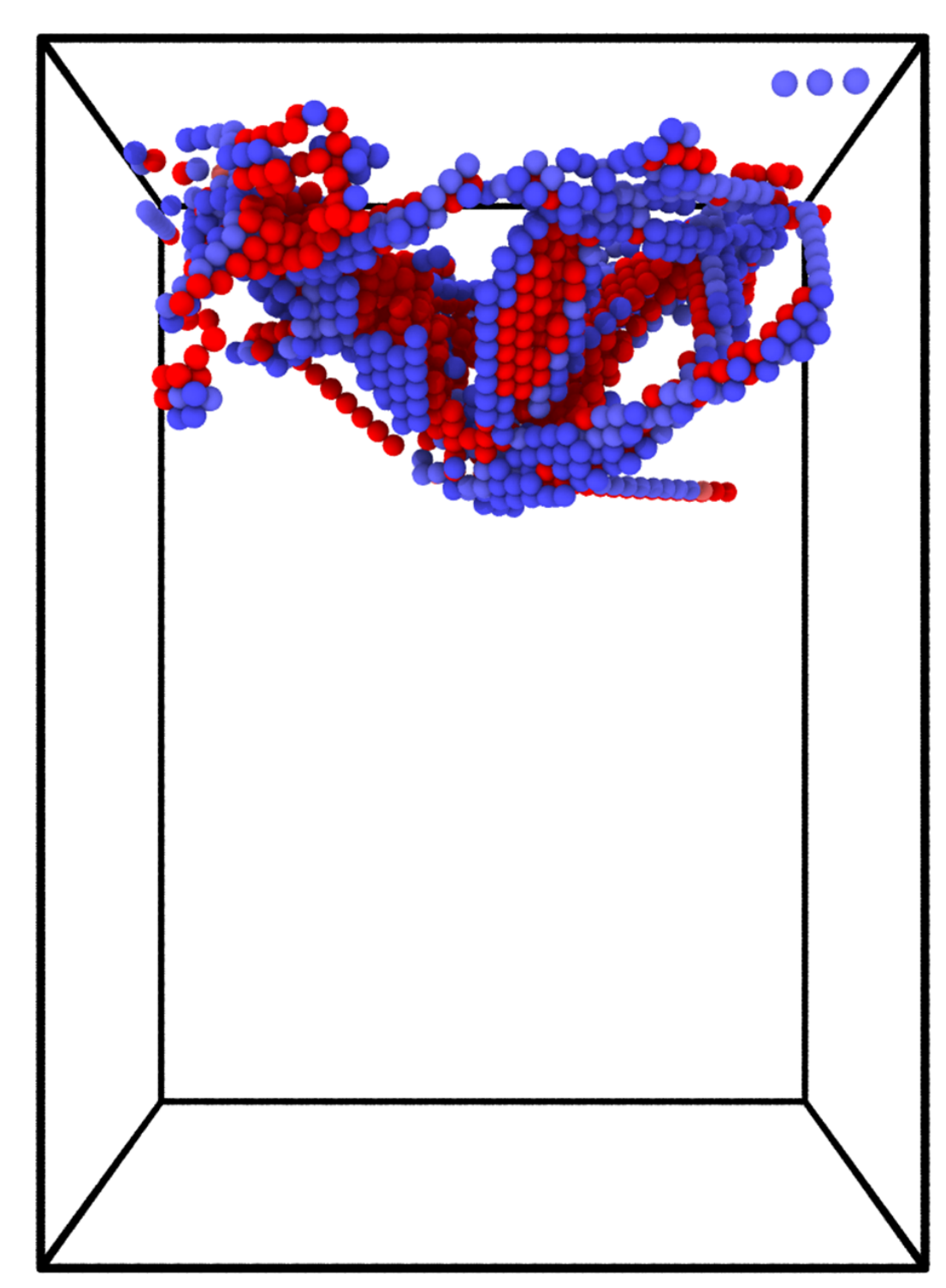

(c) 
Figure 14

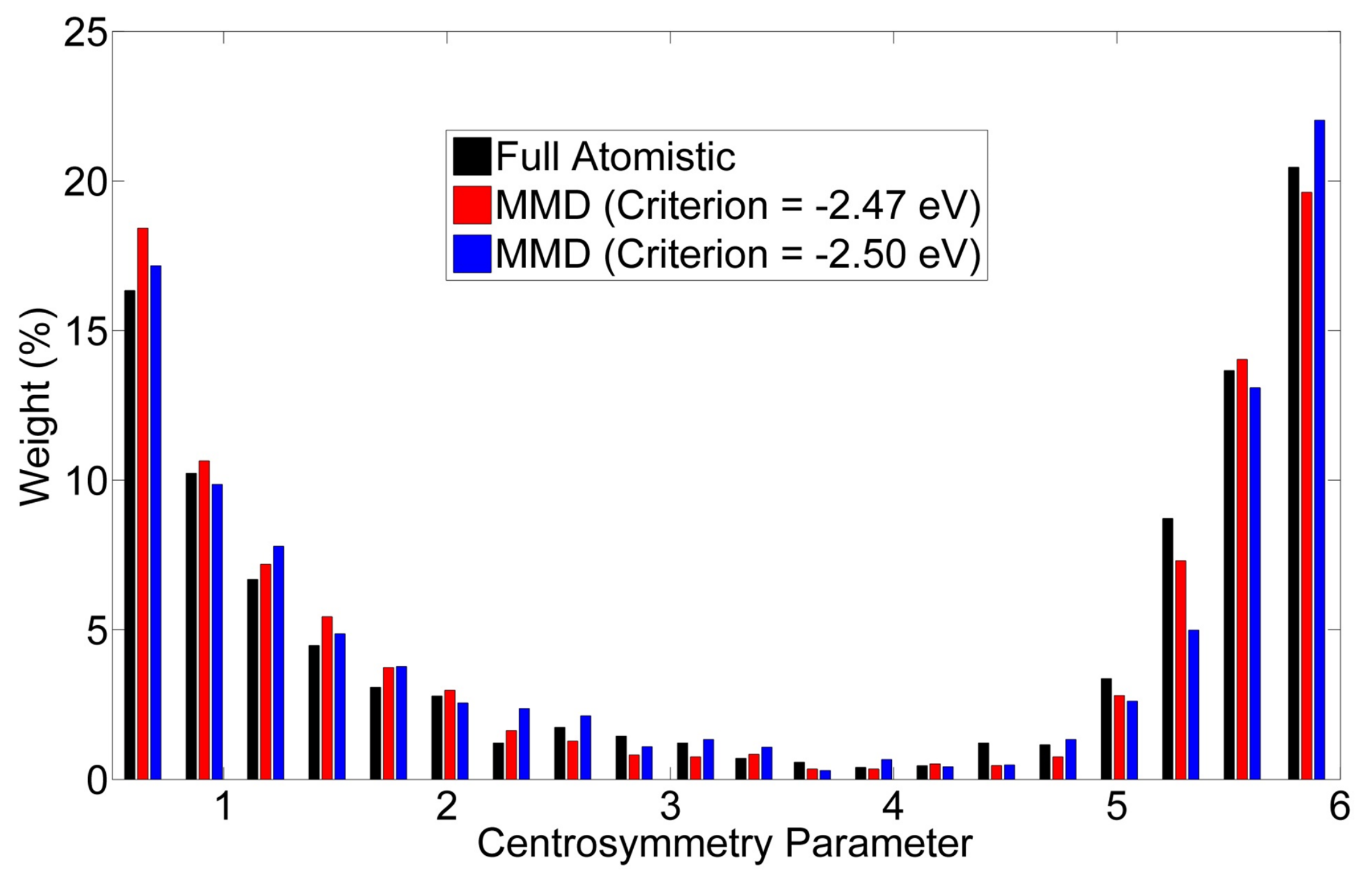


Figure 15
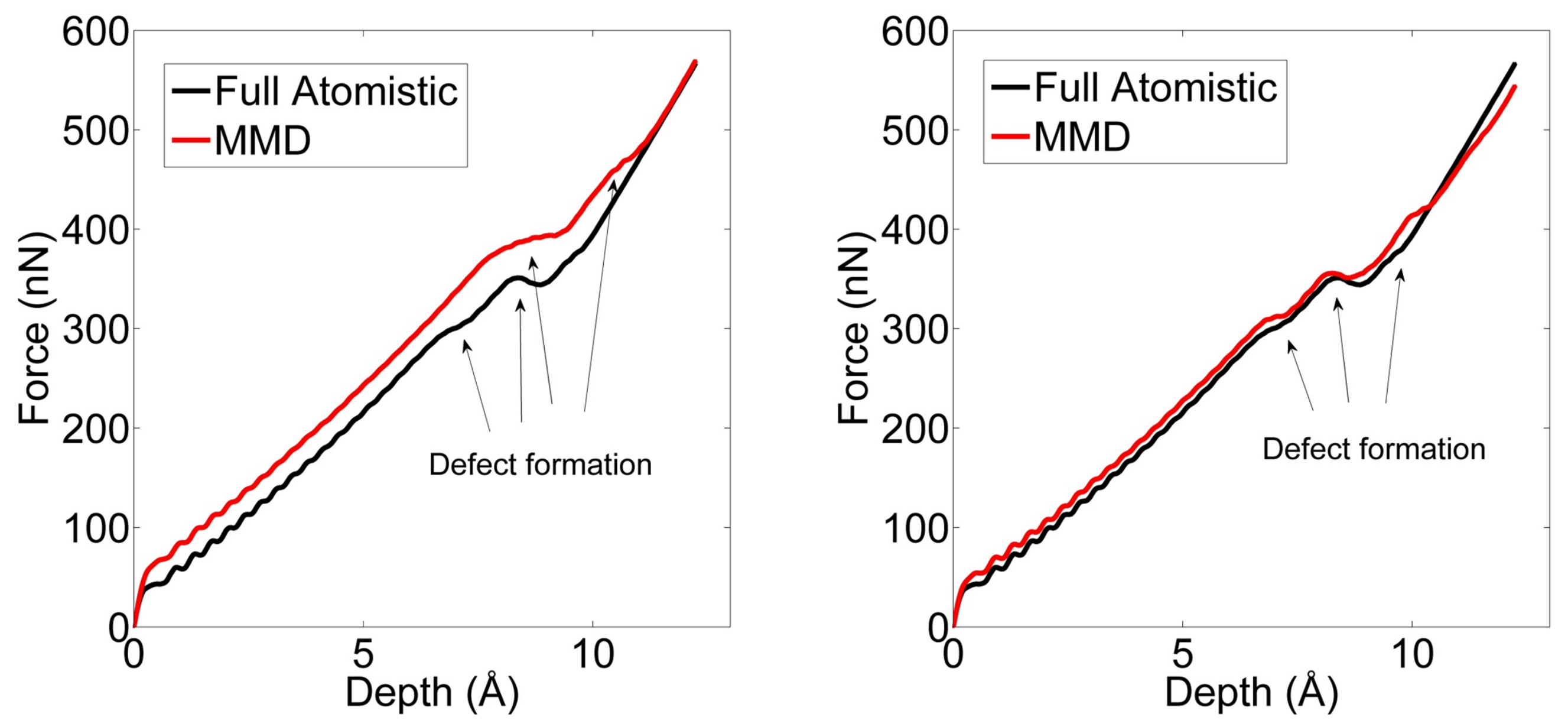
Figure 16
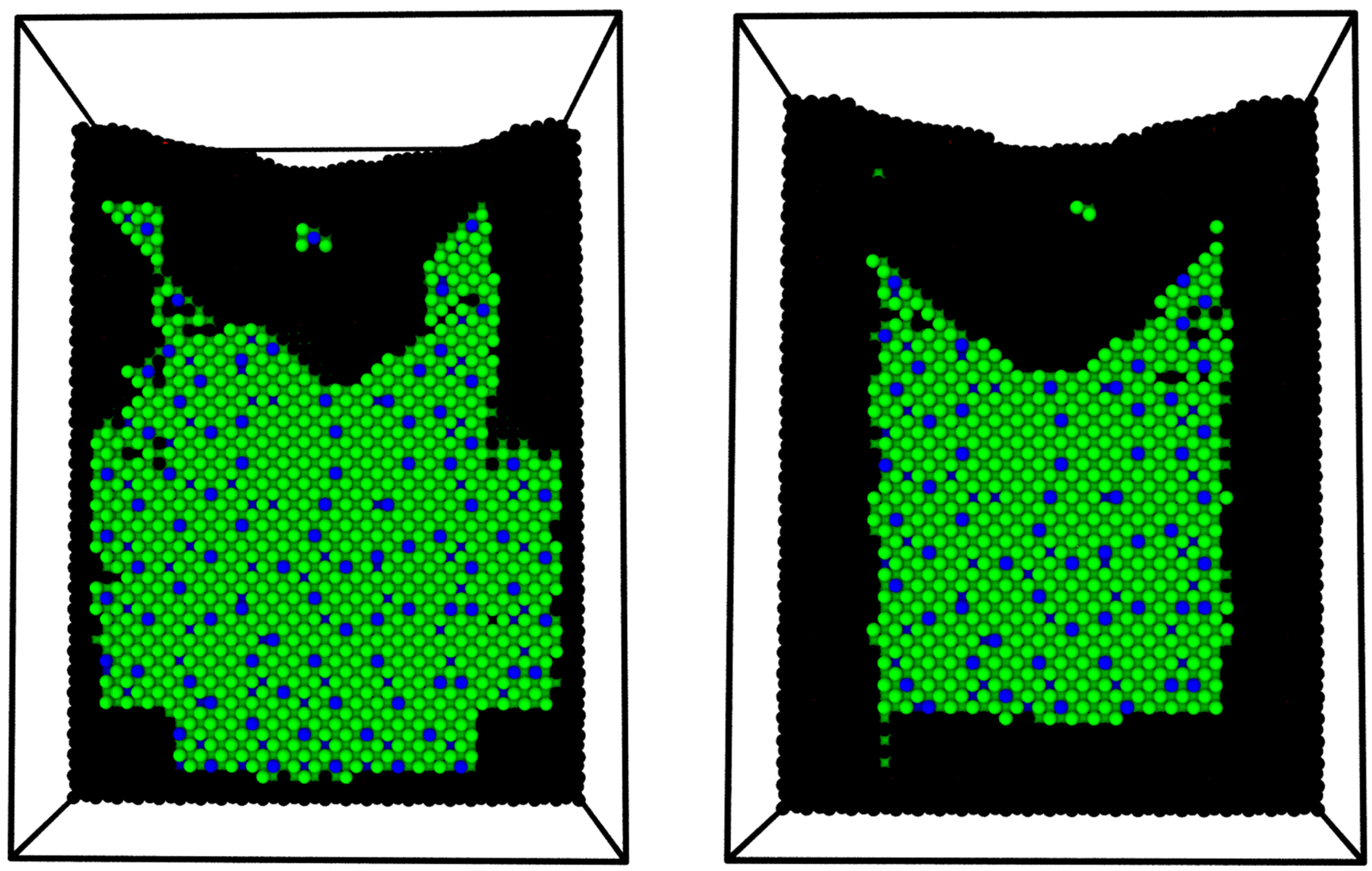